\title{
Orphans and vulnerable youth in Bulawayo, Zimbabwe: An exploratory study of psychosocial well-being and psychosocial support
}

\author{
Laelia Gilborn \\ Population Council \\ Louis Apicella \\ Population Council \\ Jonathan Brakarsh \\ Linda Dube \\ Kyle Jemison
}

See next page for additional authors

Follow this and additional works at: https://knowledgecommons.popcouncil.org/departments_sbsr-hiv

Part of the Demography, Population, and Ecology Commons, Health Policy Commons, Immune System Diseases Commons, International Public Health Commons, Maternal and Child Health Commons, Medicine and Health Commons, Public Health Education and Promotion Commons, and the Virus Diseases Commons How does access to this work benefit you? Let us know!

\section{Recommended Citation}

Gilborn, Laelia, Louis Apicella, Jonathan Brakarsh, Linda Dube, Kyle Jemison, Mark Kluckow, Tricia Smith, and Leslie M. Snider. 2006. "Orphans and vulnerable youth in Bulawayo, Zimbabwe: An exploratory study of psychosocial well-being and psychosocial support," Horizons Final Report. Washington, DC: Population Council. 


\section{Authors}

Laelia Gilborn, Louis Apicella, Jonathan Brakarsh, Linda Dube, Kyle Jemison, Mark Kluckow, Tricia Smith, and Leslie M. Snider 
Orphans and Vulnerable Youth in Bulawayo, Zimbabwe: An Exploratory Study of Psychosocial Well-being and Psychosocial Support Programs
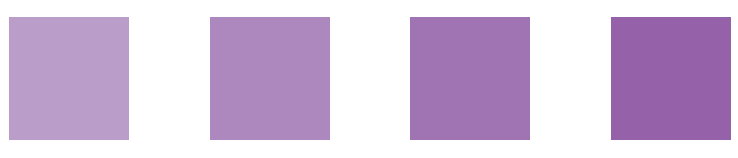

Regional Psychosocial Support Initiative Horizons Program

Catholic Relief Services/STRIVE Program 


\title{
Orphans and Vulnerable Youth in Bulawayo, Zimbabwe: An Exploratory Study of Psychosocial Well-being and Psychosocial Support Programs
}

\author{
Laelia Gilborn ${ }^{1}$, Lou Apicella ${ }^{1}$, J onathan Brakarsh ${ }^{2}$, \\ Linda Dube ${ }^{2}$, Kyle Jemison ${ }^{3}$, Mark Kluckow ${ }^{2}$, \\ Tricia Smith ${ }^{2}$, and Leslie Snider ${ }^{2}$
}

Institutional affiliations at the time of the study:

${ }^{1}$ Horizons/Population Council

${ }^{2}$ Regional Psychosocial Support I nitiative (REPSSI)

${ }^{3}$ Catholic Relief Services/STRIVE Program 


\section{Acknowledgments}

This project was a collaborative effort of the Regional Psychosocial Support Initiative (REPSSI), the Horizons Program, and the STRIVE Program ${ }^{1}$ of Catholic Relief Services (CRS). The research team at REPSSI included Linda Dube, principle investigator; Jonathan Brakarsh, Mark Kluckow, and Leslie Snider, co-principle investigators; and Tricia Smith, project coordinator. Other research team members included Laelia Gilborn of Horizons/Population Council and Kyle Jemison of CRS/STRIVE, coprinciple investigators. Lou Apicella of Horizons/Population Council managed and analyzed the data. This report was prepared by Laelia Gilborn, Mark Kluckow, Lou Apicella, Jonathan Brakarsh, Linda Dube, Kyle Jemison, Mark Kluckow, Tricia Smith, and Leslie Snider.

The authors would like to thank the communities in and around Bulawayo, Plumtree, and Zishavane, where the field work was conducted. We are also grateful to the program managers, teachers, school principals, grandmothers, and others who helped us to understand psychosocial issues among youth and to identify research respondents.

This study would not have been possible without the determination and commitment of our project coordinator, Tricia Smith. And we acknowledge with gratitude the hard work, dedication, and invaluable insights provided by our team of talented young interviewers and data entrants, some of who attended the Salvation Army Masiye Camp in their younger years. We would especially like to thank Doreen Chikume, Tobias Guzuru, Brighton Gwezera, Mandla Moyo, Doreen Muza, Beven Mwanchande, Saneliso Ndiweni, and field supervisor Karolein Vermieren.

We are indebted to our international advisory team for ensuring the quality and relevance of the research questions, methods, analysis, and this report. A special thanks to Laurie Bauman, Paul Bolton, Neil Boris, Geoff Foster, Stefan Germann, Bev Killian, Kurt Madorin, Josef Mafara, Ncazelo Ncube, Lewis Ndhlovu, and Greg Powell. Victoria James of USAID/Zimbabwe offered invaluable support and guidance as well. We also would like to acknowledge Naomi Rutenberg, Ellen Weiss, and Lisanne Brown of the Horizons Program, for providing technical comments on earlier drafts of this report.

Thanks to Ezekiel Mafusire of Salvation Army Masiye Camp and others who have made efforts to ensure that the research findings are used to improve programs. Thanks also to Noreen Huni and Miso Dhliwayo of REPSSI and Carol Hendrick of Population Council.

Finally, we owe our greatest debt to the youth who participated in this research. We sincerely hope that our collective efforts will lead to improved psychosocial support for vulnerable youth in Bulawayo and elsewhere.

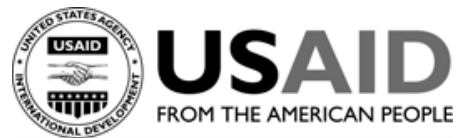

This project was funded jointly by the Horizons Program and REPSSI. Horizons is implemented by the Population Council in collaboration with the International Center for Research on Women,

International HIV/AIDS Alliance, PATH, Tulane University, Family Health International, and Johns

Hopkins University. Horizons is funded by the President's Emergency Plan for AIDS Relief through the U.S. Agency for International Development, under the terms of HRN-A-000-97-00012-00. The opinions expressed herein are those of the authors and do not necessarily reflect the views of the U.S. Agency for International Development.

\section{(P) Population Council}

The Population Council is an international, non-profit, nongovernmental institution that seeks to improve the well-being and reproductive health of current and future generations around the world and to help achieve a humane, equitable, and sustainable balance between people and resources. The Council conducts biomedical, social science, and public health research and helps build research capacities in developing countries. Established in 1952, the Council is governed by an international board of trustees. Its New York headquarters supports a global network of country offices.

Published in February 2006, Copyright 2006 The Population Council Inc.

Suggested citation: Gilborn, Laelia, Lou Apicella, Jonathan Brakarsh, Linda Dube, Kyle Jemison, Mark Kluckow, Tricia Smith, and Leslie Snider. 2006. "Orphans and vulnerable youth in Bulawayo, Zimbabwe: An exploratory study of psychosocial well-being and psychosocial support,” Horizons Final Report. Washington, DC: Population Council.

This document may be reproduced in whole or in part without permission of the Population Council provided full source citation is given and the reproduction is not for commercial purposes.

${ }^{1}$ Funded by USAID, the STRIVE Program stands for Support to Replicable, Innovative Village/Community-level Efforts. The program works with children affected by HIV/AIDS. 


\section{Table of Contents}

\section{Executive Summary}

$\begin{array}{ll}\text { Introduction } & 8\end{array}$

$\begin{array}{lr}\text { Objectives and conceptual framework } & 8\end{array}$

$\begin{array}{ll}\text { Methods } & 11\end{array}$

$\begin{array}{ll}\text { Study design } & 11\end{array}$

$\begin{array}{ll}\text { Study sample } & 11\end{array}$

$\begin{array}{ll}\text { Data analysis } & 13\end{array}$

$\begin{array}{ll}\text { Ethical procedures } & 14\end{array}$

$\begin{array}{ll}\text { Limitations of the research } & 15\end{array}$

$\begin{array}{ll}\text { Results } & 16\end{array}$

$\begin{array}{ll}\text { Profile of the sample } & 16\end{array}$

Relationships between psychosocial outcomes and respondents' sex, orphan status, $\quad 23$ and age

Associations between participation in psychosocial support interventions and 28 psychosocial outcomes

Conclusions and Recommendations 32

$\begin{array}{ll}\text { Program implications } & 33\end{array}$

Research implications $\quad 35$

$\begin{array}{ll}\text { References } & 37\end{array}$

$\begin{array}{ll}\text { Appendices } & 38\end{array}$ 



\section{Executive Summary}

Children and youth affected by AIDS typically face a wide range of stressful events and circumstances, including poverty, the loss of caregivers and loved ones, having to drop out of school, the burden of adultlike responsibilities, and social isolation. Increasingly programs for orphans and vulnerable children are addressing not only their material and educational needs, but their psychosocial needs as well. Yet there has been little research on how to evaluate psychosocial support (PSS) programs and the impact of these programs on vulnerable youth's psychosocial well-being.

This report presents findings from an exploratory study by the Regional Psychosocial Support Initiative (REPSSI) and Catholic Relief Services' Support to Replicable, Innovative Village/Community-level Efforts (STRIVE) Program of vulnerable youth living in and around Bulawayo, Zimbabwe. It describes their demographic characteristics, exposure to stress and trauma, and psychosocial well-being. The report also highlights the relationships between psychosocial well-being outcomes and exposure to stress and trauma, and the differences in psychosocial well-being between males and females, orphaned and nonorphaned youth, and younger and older adolescents. In addition, the report explores the relationships between exposure to different psychosocial support programs and measures of psychosocial well-being and distress. The report concludes with program and research implications.

\section{Methods}

First, formative qualitative research was conducted with youth and adults working with youth to determine local concepts, manifestations, and domains of well-being among youth. These findings, together with input from local youth and international research experts and psychologists, were used to draft a quantitative survey.

After pre-testing and finalizing the survey, the researchers administered it to a cross-sectional sample of 1,258 orphans and vulnerable youth, ages 14 to 20 . All of the youth fell into one of three intervention groups, or into a fourth comparison group. The intervention groups included (1) youth exposed to community PSS, (2) youth exposed to the Salvation Army Masiye Camp, a residential PSS program, and (3) youth who attended Masiye Camp and went on to become youth peer leaders. Youth in the comparison group had not been exposed to any known PSS program.

Data analysis was conducted in three stages to: (1) produce a profile of the sample, (2) determine relationships between psychosocial measures and demographic characteristics of the sample, and (3) explore associations between participation by youth in PSS interventions and psychosocial outcomes.

The cross-sectional design of the study does not allow for establishing a causal relationship between program exposure and psychosocial well-being measures. But, using multiple regression analysis, the researchers were able to explore differences in select psychosocial well-being variables (e.g., self- 


\section{Hgrizons}

confidence) and psychosocial distress variables (e.g., sadness) between the intervention groups and the comparison group.

It should be noted that at the time of data collection, Zimbabweans faced many difficulties other than HIV/AIDS, including a major food security crisis, widespread unemployment, political corruption and violence, the collapse of medical and social services, and massive inflation. Thus, in addition to the hardships caused by HIV/AIDS in their families and communities, youth in this study confronted additional stress as a result of the political and economic environment.

\section{Key Findings}

\section{Youth reported high levels of exposure to traumatic events and daily life stress.}

Most respondents had experienced multiple traumatic events, including the death(s) of loved ones, illness in the family, stigma, and rejection in times of need. Respondents also identified numerous causes of stress in their daily lives, such as lack of money, job opportunities, and health care. Zimbabwe's difficult political and economic situation contributed to these stressors faced by respondents. Another source of stress identified by many youth was not having adults to talk to about relationships, problems, and other issues, highlighting the importance of adult guidance for youth.

\section{Indications of psychosocial distress were widespread.}

More than half of the youth reported feelings of worry or stress (70 percent), irritability (65 percent), sadness (63 percent), difficulty concentrating (62 percent), being overwhelmed (61 percent), and hopelessness (56 percent) some or most of the time in the last month. These are outcomes that, in a Western context, would be suggestive of depression and anxiety.

\section{Social support for vulnerable youth was inconsistent.}

Social support questions revealed a mixed picture, with about half of youth reporting feeling "very well" supported by adults in their lives (55 percent) and people in their religious community (47 percent). However, a significant vulnerable group also emerged, who never felt supported by their community (46 percent), peers (23 percent), or people in their religious community (15 percent). Many respondents also reported abandonment by family and friends in times of need. Orphans in particular reported not having any adults to talk to about problems, and 22 percent of those living with guardians had been made to feel unwelcome. Orphans scored lower on the social support scale than did non-orphans, suggesting that orphans have less social support. 


\section{Psychosocial distress was associated with trauma, daily life stress, and lack of social support.}

Exposure to trauma and daily stress were strongly associated with depression- and anxiety-like symptoms. Greater social support, on the other hand, was negatively associated with psychosocial distress symptoms, and positively associated with some aspects of psychosocial well-being. These findings were expected and supported the measures that were chosen to assess psychosocial distress and well-being.

\section{Most youth showed signs of psychosocial well-being and resilience, but a substantial minority was particularly vulnerable.}

Despite cumulative stresses and trauma, responses indicated a good deal of resilience among the youth studied. Nearly 80 percent of the sample reported confidence in themselves, capacity to help themselves, and hopefulness for the future. The findings also suggest the existence of post-traumatic growth; greater trauma was associated with greater coping capacity among respondents. However, a very vulnerable group also emerged from questions related to resilience; nearly a third (31 percent) reported that they never feel able to cope with difficulties, 22 percent never feel hopeful about the future, and 21 percent never feel confident in themselves.

\section{Females reported more trauma, stress, and psychosocial distress than males.}

In this sample, females were more likely than males to have experienced the death of a loved one and other traumatic events and reported more sources of stress in their daily lives. They also reported markedly higher levels of psychosocial distress. For example, they reported less hopefulness and selfconfidence than males did, and were more likely to manifest this distress through somatic (physical) symptoms, such as poor appetite and fatigue.

\section{Orphanhood was associated with greater stress, isolation, and psychosocial distress.}

The experience of orphanhood was associated with greater exposure to stress, less social support, and lower levels of psychosocial well-being. For example, orphaned youth were more likely than nonorphaned peers to feel worried, irritable, overwhelmed, and to have difficulty concentrating. Compared to their peers, orphaned youth had poorer access to supportive adults in whom they could confide.

\section{Despite greater exposure to trauma and levels of psychosocial distress, older respondents showed greater signs of strength than younger respondents.}

Compared to younger youth, older respondents had higher trauma scores and exhibited more signs of psychosocial distress. For example, they were more likely to feel "alone in the world," hopeless, and 


\section{Hgrizons}

worried or stressed. At the same time, older respondents, many of whom were peer leaders, tended to report more self-confidence and feelings of self-worth than younger respondents in the sample.

\section{Psychosocial distress and well-being can co-exist.}

Within a given person, psychosocial distress was not mutually exclusive with psychosocial strength, resilience, or well-being. Growing stronger as a result of trauma does not mean that an individual does not also feel distress at the same time, or vice versa.

\section{Participation in a psychosocial support intervention was associated with greater self- confidence among males.}

Males who were in the community PSS, Salvation Army Masiye Camp, and leader groups were significantly more likely to report feelings of self-confidence relative to those in the comparison group. For females, being in the leader group was associated with greater self-confidence relative to the comparison group, but the difference was not statistically significant. Positive associations with the other psychosocial well-being outcomes-hopefulness, self-efficacy, and ability to copewere much more limited among both males and females who participated in each of the interventions.

\section{Respondents in each of the three intervention groups reported more psychosocial distress than comparison group youth.}

A greater proportion of males in the leader group reported being overwhelmed and feeling hopeless than youth in the comparison group. In addition, a greater proportion of females in the leader group reported crying, hopelessness about the future, and disinterest in life than females in the comparison group. Although none of these differences were statistically significant, the overall pattern suggests that leaders, particularly females, may have experienced greater emotional distress.

Several reasons may explain the higher levels of psychosocial distress reported by respondents in the intervention groups. One reason may be selection bias. Since communities selected the most vulnerable youth to participate in the interventions, the intervention groups may have been more vulnerable to start with than the comparison group. Multivariate regression analysis should have controlled for this, but some differences may have still emerged as a result of unmeasured factors that were also correlated with the outcomes. It could also be that the interventions encouraged youth to discuss their emotions and that as a result, participants became more emotionally expressive. But it is also possible that the programs brought out emotions without adequately helping youth to resolve them. 


\section{Program I mplications}

\section{It is necessary to find creative ways of sensitizing parents and caregivers to children's needs to talk to someone about their feelings, relationships, and healthy life decisions.}

Youth in this study were very vocal about their need for guidance and support from adults, and for greater social support in general. An important role for PSS programs would be to link youth with trusted and reliable adults with whom they can discuss issues of importance. Community and peer support for vulnerable youth should also be encouraged and developed.

PSS programs need to be aware of the potential added vulnerability of females to trauma, daily stress, and psychosocial distress.

It may be necessary to strengthen the ability of programs to recognize and address problems and issues that particularly affect females, such as poor self-esteem. Programs should learn to recognize physical complaints as possible indications of psychosocial distress. Some "girls only" activities may be useful to address their special needs and vulnerabilities.

\section{Older youth may benefit from tailored PSS programs that help them cope with increasing responsibilities.}

With age comes mounting pressure and stress, as well as increased exposure to trauma. To help cope with unemployment and relationship issues, PSS programs should develop ways to link older youth to adult mentors and social support services.

\section{PSS programs need to be responsive to the unique needs of orphaned adolescents without causing further stigmatization.}

In comparison to their non-orphaned peers, orphaned (maternal, paternal, and double) adolescents demonstrated greater psychosocial distress. Programs must respond to the strains of parental illness and death on adolescents, but be cautious about targeting orphaned youth lest they further stigmatize them or neglect the needs of other vulnerable youth.

\section{PSS programs should explore better ways to address psychosocial distress on the one hand,} while cultivating resilience on the other.

Psychosocial distress was widespread among vulnerable youth in the study area. But youth also drew on their own constructive coping skills, and most demonstrated a degree of confidence in their ability to manage life stressors. Since psychosocial well-being and psychosocial distress are not mutually exclusive, 


\section{Hgrizons}

programs must both promote psychosocial well-being and reduce psychosocial distress and grief. Programs should aim to ensure that the activities do not simply bring out emotions and encourage youth to express them in a supportive environment, but also help youth to cope with feelings, such as sadness, over time. PSS programs must be sensitive to each child's particular needs (such as children who require longer-term or more skilled support in grief resolution, protection from abuse, and so forth) and should incorporate ongoing, community-based follow-up.

\section{PSS programs should be adapted to reflect children's grieving processes and attitudes toward illness, loss, and death in this specific cultural context.}

Grief counseling and its perceived emotional benefits to orphans and vulnerable children and youth is central to the Salvation Army Masiye Camp model, but may need to be revisited. An approach that takes a longer-term perspective to the grieving process and supports children appropriately at the various stages of that process may prove more beneficial.

\section{A comprehensive response to the psychosocial needs of vulnerable youth must go beyond discrete PSS programs to address the underlying contextual causes of trauma and stress.}

Adolescents in the study confronted many traumatic experiences and high levels of day-to-day stress. This context represents a serious challenge for PSS programs. PSS can comfort, support, and guide youth, and can encourage confidence, self-esteem, and coping skills. However it may not be realistic to expect the types of PSS programming examined in this study to greatly reduce psychosocial distress in the presence of multiple and pervasive causes of trauma and stress in this setting. Therefore, broader program and policy responses to address economic and sociocultural factors contributing to trauma and stress are needed as well.

\section{Research I mplications}

\section{The survey instrument needs to be tested for validity and reliability.}

The study's attempts to measure psychosocial well-being and distress were supported by the expected associations found between stress and trauma and psychosocial distress, and the associations between social support and psychosocial well-being. However the instrument should be further tested to ensure its validity and reliability in measuring psychosocial well-being, emotional distress, and grief among youth in Zimbabwe; its usefulness in other country contexts, cultures, and languages; and its ability to capture psychosocial issues relevant to youth of both sexes and different ages. 


\section{Further research is needed to see what factors are associated with resilience and post- traumatic growth.}

Despite widespread trauma, daily stress, and psychosocial distress, many youth maintained selfconfidence, hopefulness, and social connectedness.

\section{In-depth qualitative research is needed to better understand children's grieving processes.}

Such research would focus on cultural attitudes, and children's attitudes in particular, toward illness, loss, and death. The findings would be useful for PSS programs working with children on grief resolution.

\section{Quasi-experiments should be conducted to more rigorously assess the impact of PSS programs.}

This study was exploratory; a more rigorous study would feature a pre-post design. Ideally, postintervention observations would be conducted with youth participants soon after the intervention is completed and again after some time has lapsed to determine whether any impact on psychosocial wellbeing is sustained over time. Future research should also isolate the effects of different intervention components to determine how activities can be improved. 


\section{Hgrizons}

\section{I ntroduction}

Children and youth affected by AIDS typically face a wide range of stressful events and circumstances, including poverty, the loss of caregivers and loved ones, having to drop out of school, the burden of adultlike responsibilities, and social isolation. Increasingly programs for orphans and vulnerable children and youth are addressing not only their material and educational needs, but also their psychosocial needs. Yet there has been little research on how to evaluate psychosocial support programs and on assessing the impact of these programs on vulnerable youth's psychosocial well-being.

In 2003 Horizons began a research collaboration with the Regional Psychosocial Support Initiative (REPSSI) and Catholic Relief Services' Support to Replicable, Innovative Village/Community-level Efforts (STRIVE) Program to respond to these knowledge gaps. This report presents findings from an exploratory study of 1,258 vulnerable youth, ages 14 to 20. It describes their demographic characteristics, exposure to stress and trauma, and psychosocial well-being. The report also highlights the relationships between psychosocial well-being outcomes and exposure to stress and trauma, and explores the differences in psychosocial well-being between males and females, orphaned and non-orphaned youth, and younger and older adolescents. In addition, the report examines the relationships between exposure to different psychosocial support programs and measures of psychosocial well-being and distress. The report concludes with program and research implications.

It should be noted that at the time of data collection, Zimbabweans faced many difficulties other than HIV/AIDS, including a major food security crisis, widespread unemployment, political corruption and violence, the collapse of medical and social services, and massive inflation. Thus, in addition to the hardships caused by HIV/AIDS in their families and communities, youth in this study confronted additional stress as a result of the political and economic environment.

\section{Objectives and Conceptual Framework}

The study objectives were to:

- Develop a better understanding of psychosocial well-being among vulnerable adolescents and how to measure it.

- Explore the effects of participation in psychosocial support programs on adolescents' psychosocial well-being.

The study focuses on adolescents because of the presence of psychosocial support programs for this age group in the study area. Adolescence is a distinct stage in psychological development, and vulnerable adolescents, especially those affected by HIV/AIDS, face unique responsibilities and challenges, such as earning wages, managing households, and caring for younger children and sick adults.

In this study, a person's psychosocial well-being (PSWB) refers to his/her intrapersonal (i.e. internal) emotional and mental state (psycho-) and his/her interpersonal network of human relationships and social connections and functioning (-social). These two aspects of well-being are interrelated. Good or high 
psychosocial well-being is when one's mental/emotional state and social relationships are predominantly positive, healthy, and adaptive. Poor psychosocial well-being or psychosocial distress is when these are mostly negative, unhealthy, or maladaptive. The concepts of psychosocial well-being and distress must be understood according to the sociocultural context, and the age and developmental stage of the child.

Psychosocial support (PSS) includes formal and informal services that address psychosocial well-being either (1) directly and specifically (e.g., through interpersonal moral support, counseling, spiritual support, creation of memory books, etc.) or (2) indirectly (e.g., school and nutritional support programs that may alleviate stress and worry). This study focuses on programs that target PSWB directly and explicitly.

The conceptual framework for this study (Figure 1) posits that PSWB is influenced by (1) individual factors and experiences (such as age, sex, innate personality characteristics, family or household structure, personal exposure to stress and trauma, and socioeconomic status), (2) contextual factors (e.g., community cohesiveness and support, presence of AIDS-related stigma, the sociopolitical environment, and availability of educational and employment opportunities), and (3) access to programs and services, including PSS programs. This study explored the relationships between PSWB and individual factors and experiences, and participation in PSS programs.

The framework also posits that these are two-way relationships. Individual factors and experiences, contextual factors, and access to programs and services can influence a person's psychosocial well-being and vice versa. For example, experiences of trauma or living in an unsupportive community can have a detrimental effect on one's psychosocial well-being. A hopeful, self-confident person might be more able to overcome stressful and traumatic events or improve their socioeconomic status by obtaining education or employment. Also one's psychosocial well-being may influence the degree to which one is "capable" of benefiting from PSS. For example, someone with high self-esteem might be quicker to get involved with experiential learning. On the other hand, a person with low self-esteem might have more to gain from PSS. 


\section{Hgrizons}

Figure 1 Conceptual framework

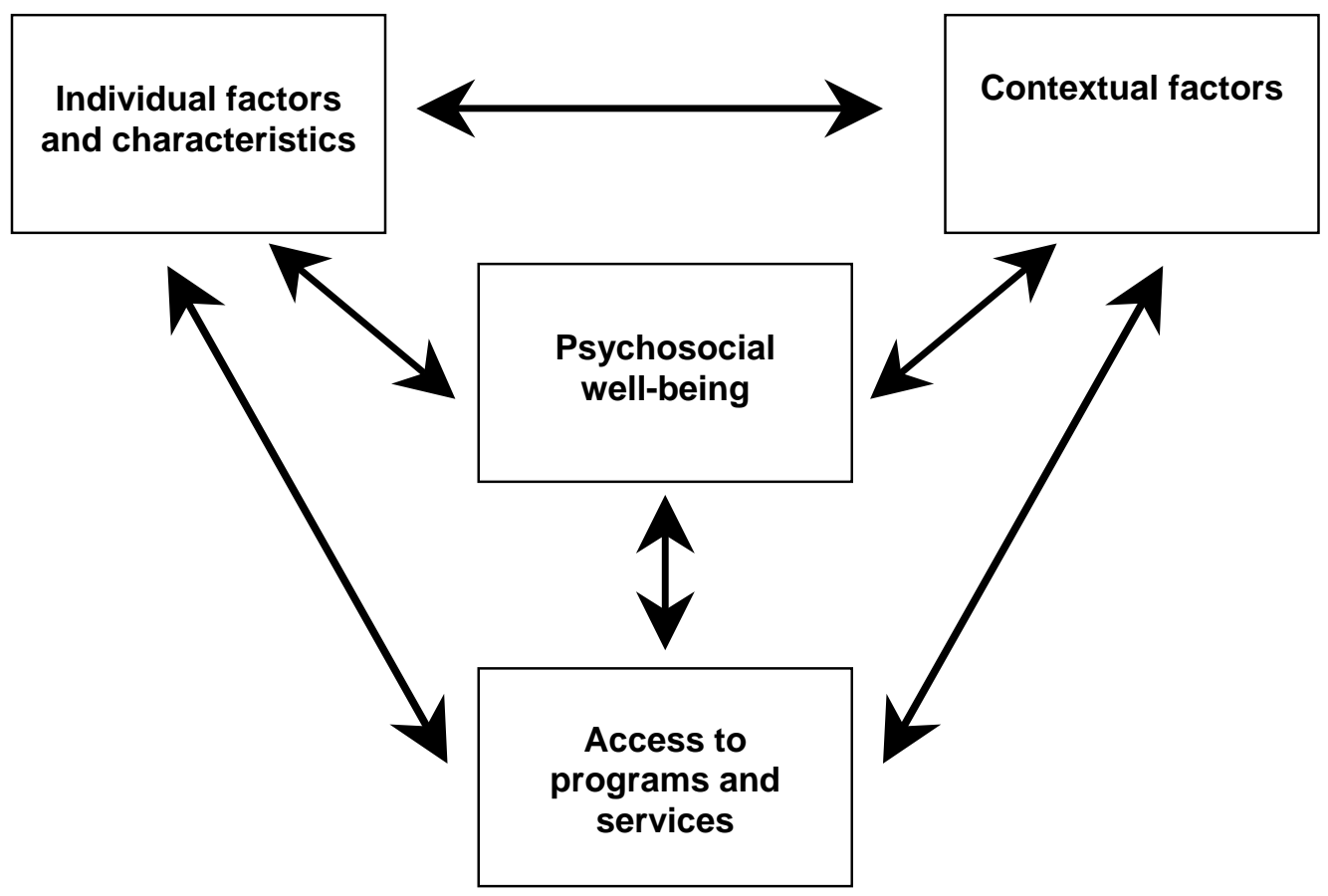




\section{Methods}

\section{Study Design}

This exploratory study consisted of two phases of data collection: formative qualitative research and a cross-sectional survey.

In an effort to identify local concepts and manifestations of psychosocial well-being and distress among youth, researchers conducted formative, qualitative research in June 2003 in the Bulawayo area in Zimbabwe. Informants included youth and adults working with youth, such as teachers, NGO staff, pastors, and counselors. Data collection methods included in-depth interviews and focus groups, followed by pile-sort exercises to identify domains of psychosocial well-being.

Researchers then used qualitative findings and select items from standardized surveys (developed in Western settings but applied previously in Africa) to draft a survey instrument to assess psychosocial well-being among vulnerable youth. The draft survey tool was refined, based on pre-testing and feedback from local youth and international experts.

The revised survey was administered to a sample of 1,258 vulnerable youth living in 32 communities in and around Bulawayo during the period between September 2003 and February 2004. The interviews took about 45 minutes to complete, and were administered by same sex, same language interviewers whenever possible.

The survey elicited information about respondents' demographic characteristics, exposure to stress and trauma, orphan status, and perceived social support. The survey also included variables to assess psychosocial well-being (e.g., self-confidence) and psychosocial distress (e.g., sadness). The items can be found in Appendix A (page 38).

\section{Study Sample}

All youth in the study sample fit into one of four study groups (Table 1). The first group consisted of youth who participated in community PSS programs. In Bulawayo and throughout Zimbabwe, the STRIVE Project supports faith-based and other community groups to offer structured, specialized activities to foster ongoing, sustainable support to orphans and vulnerable children. The community PSS programs take many forms, including training teachers to recognize and respond to psychosocial distress among students; Kids' Clubs which meet weekly for group recreation, socialization, and to play games that address issues important to children and youth; and group and individual counseling and problemsolving.

The second group was comprised of youth who attended the Salvation Army Masiye Camp, a residential, faith-based program for vulnerable children and youth in Matabeleland Province near 


\section{Hgrizons}

Matopos National Park. Camp sessions usually last 10 days and are explicitly designed to improve the psychosocial well-being of participants. This program encourages personal growth in areas such as selfesteem, trust, effective communication, conflict resolution, problem-solving, and grief resolution. Camp activities emphasize adventure-based, experiential learning that creates opportunities for discussing life challenges and problem solving. Contact with counselors gives participants an opportunity to talk about how they feel about their experiences and losses in a safe environment. Spiritual practice is also emphasized as a means of coping with loss and maintaining hopefulness. The intention is for all children to be linked with community organizations for follow-up support, although actual levels of follow-up support are inconsistent.

Embedded within both Masiye Camp and community PSS programs are opportunities for youth to serve as mentors and leaders to other vulnerable youth. Thus the leader group consists of youth who attended Masiye Camp as campers and then became either Masiye Camp counselors or community PSS youth leaders. Some youth in this group are responsible for leading Kids' Clubs for younger vulnerable children. Others work as counselors at Masiye Camp or as AIDS peer educators. While these peer leadership programs are designed first and foremost to support, motivate, and inspire orphans and vulnerable children, peer leadership programs may also foster psychosocial well-being in the leaders themselves through training, camaraderie, and the experience of serving as a role model and counselor.

The fourth group, or comparison group, consisted of youth ages 14 to 20 living in the same communities as the other study groups. These youth were identified by key informants and community members as being vulnerable (e.g., having one or more parents ill or deceased, living in extreme poverty) and never having participated in any formal, direct psychosocial support program. ${ }^{2}$

Table 1 Sample size by sex and study group

\begin{tabular}{lccc}
\hline & Males & Females & Total \\
\hline Community PSS group: exposed to community PSS only & 149 & 191 & 340 \\
Camp group: exposed to Masiye Camp only & 133 & 115 & 248 \\
Leader group: Masiye Camp + became a counselor or leader & 55 & 57 & 112 \\
Comparison group: exposed to none of the above & 267 & 291 & 558 \\
Total sample & 604 & 654 & 1,258 \\
\hline
\end{tabular}

Initially, respondents in each of the three intervention groups were recruited from program databases of past participants. All those who were between the ages of 14 and 20 and had participated in the programs within the past two years were invited to participate. The databases did not yield enough respondents for the sample size needed to examine differences between groups. The numbers were therefore

\footnotetext{
${ }^{2}$ REPSSI has committed to ensuring that after the study, all children from the comparison group will receive PSS through either community-based initiatives or the Masiye Camp.
} 
supplemented by asking community members to identify youth program participants. The sample includes respondents from 32 districts in four provinces in Zimbabwe: Matebeleland South, Bulawayo, Midlands, and Matebeleland North.

\section{Data Analysis}

Analysis was conducted in three stages and the results presented accordingly.

1) Profile of the sample:

Survey data were entered in EpiInfo Version 3.3 and analyzed in SPSS, version 12.0.2. Frequencies were run in order to generate a profile of the study population with respect to demographics, exposure to stress and trauma, social support, orphan status, psychosocial well-being, psychosocial distress, and other characteristics.

Four count variables were created to capture individual factors and experiences that affect psychosocial well-being: (1) exposure to traumatic events (trauma score), (2) stress in daily life (daily stress score), (3) social support (social support score), and (4) personal possessions (a rough proxy for socioeconomic status). For details on how these composite index variables were created, refer to Appendix B (page 39).

2) Relationships between psychosocial outcomes and demographic characteristics:

Differences in psychosocial measures (exposure to stress and trauma, social support, psychosocial wellbeing, and psychosocial distress) by sex, orphan status, and age of respondents were explored. Pearson's two-sided chi-square test for general association was chosen to evaluate significance in relationships. The Student's T-test was used to compare sample means by calculating the two-tailed probability of the difference between the means. A p-value of less than 0.05 was used as a threshold for significance for all tests.

3) Associations between participation in psychosocial support interventions and psychosocial outcomes: In exploring the effects of each program, the researchers focused on a subset of 12 psychosocial outcomes clustered into three broad domains (Table 2). The effects of each program were assessed separately by comparing these outcomes among respondents in each intervention group to respondents in the comparison group. Appendix C (page 40) describes the regression methods used for this analysis.

Table 2 Psychosocial domains and outcomes

\begin{tabular}{lll}
\hline Psychosocial well-being & Psychosocial distress & Lingering grief \\
\hline - Self-confidence & - Sadness & - Sadness about deaths \\
- Hopefulness & - Crying & - Anger about deaths \\
- Self-efficacy & - Feeling overwhelmed & - Fear about deaths \\
- Ability to cope & - Hopelessness & \\
& - Disinterest in life & \\
\hline
\end{tabular}




\section{Hgrizons}

Regression analysis was used to control for other individual and contextual factors, aside from exposure to the PSS programs, which influence psychosocial outcomes (e.g., age category, province, type of setting, possessions score, trauma score, daily stress score, and social support score). Again, a level of $\mathrm{p}<0.05$ for the adjusted odds ratio is considered statistically significant

Analyses showed significant differences between males and females with respect to many of the psychosocial outcome measures (Table 9, page 23). Therefore, regression analysis was conducted separately for males and females, and the results are presented accordingly.

\section{Ethical Procedures}

The research protocol was reviewed and approved by the Population Council's Institutional Review Board. The study's international advisory team and local youth on the research team also reviewed the research instruments and procedures and provided guidance.

Questionnaires were reviewed and revised to eliminate unnecessarily upsetting or disturbing interview questions. The research team was comprised largely of young people with similar experiences to those of the respondents. Zimbabwean psychologist Mark Kluckow (one of the principle investigators) trained the interviewers to pause and support respondents if interviews provoked a strong emotional reaction and to continue with the interview only if and when the respondent agreed. They were also taught to flag serious crises, such as suicidal ideation or a history of abuse, for the supervisor to follow-up. As needed, respondents were provided with additional on-the-spot support from trained counselors on the research team and/or referred by the team supervisor for additional external support.

Although these procedures seemed to be generally sufficient, there were challenges. The team did its best to provide referrals, but services for youth dealing with serious problems such as abuse were extremely limited in the Matabeleland region at the time of the study. Some issues were difficult to follow-up without violating confidentiality and/or putting the respondents at risk. One advisor to the study suggested that in future research, the team link with the Department of Social Welfare in order for social workers to learn about issues facing youth and as a means of more effectively linking respondents to social services. In the absence of such services interviewers were trained to help respondents to identify adults in their communities who might be of help, such as headmasters, teachers, and grandmothers.

Because the research was a retrospective cross-sectional study, there was no need to withhold services from any youth for the sake of maintaining a comparison group. Instead, the team simply identified youth who had not received PSS services in the past. Furthermore, all participants in the comparison group were later offered the opportunity to attend a community-based PSS activity, with the result that the research in this way became a direct gateway to PSS services for these youth. However, to avoid putting pressure on individuals to participate in the study, comparison group respondents were not informed of this arrangement until the survey was complete. 


\section{Limitations of the Research}

Limitations of the study include the lack of a pre-intervention survey and the fact that respondents were not randomly assigned to study groups. There was no practical or ethical way to randomize youth into intervention groups. And while a pre-post study design would have been more rigorous for determining the outcomes of the interventions, the researchers opted for a rapid cross-sectional approach. This was because the study was exploratory and the methods developed to measure psychosocial well-being had not yet been thoroughly evaluated in this setting for validity and reliability. It is important to keep in mind when reviewing the data that the cross-sectional design does not allow for establishing a causal relationship between program exposure and psychosocial well-being measures.

Because the communities identified youth to participate in PSS programs on the basis of those youth being "vulnerable" in some way, the intervention study groups may have been inherently different from the comparison group (i.e. facing greater trauma and daily stress). That being said, the research team did try to recruit similar youth for the comparison group. The leader group, in particular, likely differed from the comparison group. Youth leaders were motivated to become counselors and in many cases were seen by adults, such as Masiye Camp staff, as already having leadership qualities for which they were recruited into the counselor program. The leaders group was also older on average than the other study groups. Regression analysis was used to try to correct for these sources of bias. 


\section{Hgrizons}

\section{Results}

\section{Profile of the Sample}

A total of 1,258 youth (604 males and 654 females) were interviewed for this study. As shown in Table 3, the average age of the sample was 15.9 (age range 14 to 20). Slightly more than half were female. The majority were Christian and Ndebele speakers, reflecting the dominant religious and linguistic groups in the study area. A high percentage (83 percent) was enrolled in school, but two-thirds had not completed the expected level of school for their age. Eleven percent stated that they were working for wages.

At the time of the survey, 22 percent were double orphans (both parents deceased), more than a third were paternal orphans (father deceased), and nine percent were maternal orphans (mother deceased). Thus, about two-thirds (65 percent) had lost one or both parents. Forty-five percent lived in an urban area, with the remainder of respondents divided between peri-urban and rural settings. Almost all respondents lived in a home; four percent lived in institutions and only five respondents reported being homeless at the time of the interview.

Respondents' households had, on average, one wage earner for every five household members. But, nearly one-third of respondents (31 percent) lived in households with no wage earners. Three-quarters of respondents reported that they ate three meals a day, 23 percent ate two per day, and two percent only ate one meal per day. In terms of personal possessions, 95 percent reported that they had a blanket, 88 percent had a spare set of clothes, and 79 percent had a pair of shoes.

\section{Most respondents experienced multiple traumatic events.}

Given the cumulative impacts of death and loss on the psychological health of young people, respondents were asked about the loss of parents and other persons close to them. Almost all respondents (93 percent) had experienced the death of a loved one, with more than half having experienced multiple losses. Respondents were also asked about their exposure to other potentially traumatizing events. Illness in the family, stigma, and social isolation from relatives and friends were the most prevalent forms of trauma, as shown in Table 4.

In a separate self-completed questionnaire, respondents reported their experiences of sexual abuse. Eight percent reported molestation and six percent reported rape. These figures were similar for males and females.

A count variable captured the level of potentially traumatizing events experienced by each respondent. These events were weighted and included sexual abuse and other items (see Appendix B, page 39, for an explanation of how the trauma score was created). The range of the trauma score was from 0 to 14 , with 14 indicating the maximum number of traumatic events. The average trauma score was 5.5 (range:0-12), suggesting that most respondents had experienced multiple traumas, with potentially negative impacts on their psychological health and functioning. 
Table 3 Demographic characteristics of the sample by group

\begin{tabular}{|c|c|c|c|c|c|}
\hline & $\begin{array}{c}\text { Comparison } \\
\text { group } \\
(n=558)\end{array}$ & $\begin{array}{c}\text { Camp only } \\
\text { group } \\
(n=248)\end{array}$ & $\begin{array}{l}\text { Leader } \\
\text { group } \\
(n=112)\end{array}$ & $\begin{array}{l}\text { Community } \\
\text { PSS group } \\
(n=340)\end{array}$ & $\begin{array}{c}\text { Total } \\
(n=1,258)\end{array}$ \\
\hline \multirow[t]{2}{*}{$\overline{\text { Average age }}$} & 15.5 years & 16.0 years & 17.5 years & 15.9 years & 15.9 years \\
\hline & $\%$ & $\%$ & $\%$ & $\%$ & $\%$ \\
\hline \multicolumn{6}{|l|}{ Sex } \\
\hline Male & 48 & 54 & 49 & 44 & 48 \\
\hline Female & 52 & 46 & 51 & 56 & 52 \\
\hline \multicolumn{6}{|l|}{ Native language* $^{*}$} \\
\hline Ndebele & 76 & 85 & 81 & 52 & 72 \\
\hline Shona & 21 & 12 & 16 & 45 & 25 \\
\hline Other & 3 & 3 & 4 & 4 & 3 \\
\hline \multicolumn{6}{|l|}{ Religion } \\
\hline Christian & 92 & 96 & 95 & 97 & 95 \\
\hline Traditional & 3 & 0 & 1 & 2 & 2 \\
\hline None & 5 & 3 & 5 & 1 & 4 \\
\hline \multicolumn{6}{|l|}{$\begin{array}{l}\text { Survival status of } \\
\text { parents }^{\star^{\mathrm{a}}}\end{array}$} \\
\hline Both parents alive & 36 & 19 & 36 & 43 & 35 \\
\hline Mother deceased & 8 & 13 & 7 & 11 & 9 \\
\hline Father deceased & 33 & 43 & 38 & 27 & 34 \\
\hline Both parents deceased & 23 & 25 & 19 & 19 & 22 \\
\hline \multicolumn{6}{|l|}{ Type of community* } \\
\hline Urban & 32 & 63 & 88 & 41 & 45 \\
\hline Peri-urban & 41 & 7 & 5 & 23 & 26 \\
\hline Rural & 27 & 30 & 7 & 36 & 29 \\
\hline \multicolumn{6}{|l|}{ Education* } \\
\hline Currently in school & 94 & 71 & 49 & 86 & 83 \\
\hline \multicolumn{6}{|l|}{ Employment* } \\
\hline Currently employed & 9 & 15 & 21 & 10 & 11 \\
\hline
\end{tabular}

${ }^{*} \mathrm{p}<0.05$ (Chi-square)

a If a respondent did not know whether a given parent was alive, that parent was treated as deceased 


\section{Hgrizons}

Table 4 Most common traumatic life experiences

\begin{tabular}{ll}
\hline & $\%$ \\
\hline Death of a loved one & 93 \\
Illness in their family or household & 80 \\
People saying bad things about them or avoiding them because of their family's situation & 52 \\
Being rejected, neglected, or abandoned by their relatives when they needed them & 51 \\
Losing the support of their friends when they needed them & 49 \\
Losing family land or possessions & 45 \\
Having too much responsibility or work & 37 \\
Having to leave school when they did not want to & 18 \\
\hline
\end{tabular}

\section{Lack of social support by family, friends, and community members was common.}

Social connectedness, such as having supportive relationships with primary caregivers and members of one's cultural or faith groups, and access to community resources are widely recognized as protective factors that "buffer the consequences of negative experiences on children" (Duncan and Arnston 2003).

Participants in formative research emphasized the need to talk with adults about concerns and questions about relationships in their lives. However, they felt that adults did not acknowledge the challenges their generation currently faces in dealing with HIV/AIDS, and they felt chided or belittled when trying to discuss romantic relationships. In some cases, family disputes (e.g., about property) alienated them from the adult relative they would normally go to for advice. Quantitative data confirm that many youth do not receive the social support needed from peers and adults to mitigate the impacts of stressful and traumatic events in their lives (Table 5).

Although roughly half of respondents reported that they felt very well supported by the adults in their lives and very well supported by their religious communities, about half of respondents experienced abandonment by family members and friends during times of need. Of those respondents who had lost a parent, over one-third (38 percent, $n=789$ ) reported that they do not have anyone to talk to when they experience strong feelings about their loss(es). About a fourth (23 percent) of those living with someone other than their biological parent(s) $(n=521)$ reported being made to feel unwelcome at home. Although most youth in this sample enjoyed spending time with friends (even many of those who demonstrated signs of depression and hopelessness), only 24 percent felt very well supported by their peers. It is particularly disturbing that 46 percent of youth said that they almost never felt supported by the broader community.

A social support score was created by adding up the types and level of support from each of these groups: adults, peers, community, and religious community. (See Appendix B, page 39, for an explanation of how the social support score was created.) The average score was 8.0, based on a range of possible scores from 0 to 12 , with 12 indicating maximum social support. 
Table 5 Social connectedness

\begin{tabular}{lccc}
\hline $\begin{array}{l}\text { "How well do you feel supported by each of these } \\
\text { groups?" }\end{array}$ & $\begin{array}{c}\text { Almost } \\
\text { never } \\
\text { (\%) }\end{array}$ & $\begin{array}{c}\text { Sometimes } \\
\text { (\%) }\end{array}$ & $\begin{array}{c}\text { Very well } \\
\text { (\%) }\end{array}$ \\
\hline The adults in your life & 5 & 40 & 55 \\
Your peers & 23 & 53 & 24 \\
People in your community & 46 & 42 & 13 \\
People in your religious community* & 15 & 38 & 4 \\
\hline
\end{tabular}

*This question asked of the 954 (76\%) who regularly attended church, mosque, etc.

\section{Lack of money and job opportunities were perceived as major problems by most respondents.}

Respondents were asked about a range of potential stressors in their daily lives. The issues most commonly reported as "a big problem" are listed in Table 6 . Not surprisingly given the difficult socioeconomic and political situation at the time of the study, not having enough money or job opportunities are at the top of the list. Unemployment emerged as a very critical problem, especially for males, in the qualitative research. Indeed some youth, even those in school, saw limited benefit to getting an education, as there would be no job opportunities for them after graduation. They spoke of peers who dropped out of school to engage in illegal wage-earning activities such as panning for gold.

Survey respondents also lacked access to essential resources, including health care, social services, and information. Lack of support and guidance from adults also emerged as a significant issue for youth and echoed the previous section on social connectedness.

A daily stress score was developed to capture the number of stressors in daily life reported by each respondent (see Appendix B, page 39). The range was from 0 to 10, with 10 indicating the maximum number of stressors. The average daily stress score was 4.6, suggesting that most respondents had numerous sources of stress in daily life-a potential source of psychosocial distress.

\section{Youth reported high levels of psychosocial distress.}

Responses to various questions indicate a high level of distress among respondents (Table 7). For example, more than three-fourths of youth felt guilty some or most of the time in the past month for bad things that have happened in their life. Seventy percent felt worried or stressed and more than 60 percent felt irritable, sad, had difficulty concentrating, or felt overwhelmed. More than half felt hopeless about the future. Poor concentration, guilty feelings, and irritability are symptoms often related to depressive disorders. Headaches, nightmares, and fatigue were also commonly reported, and may reflect physical manifestations (somatization) of emotional distress. 


\section{Hgrizons}

Table 6 Stressful issues in daily life (\%)

\begin{tabular}{ll}
\hline & $\%$ \\
\hline Not enough money & 58 \\
Lack of job opportunities & 54 \\
Not enough access to health care & 46 \\
Not having adults to talk to & 45 \\
Not enough access to social services & 41 \\
Lack of safety and security & 40 \\
Lack of information needed to make decisions & 38 \\
Lack of opportunities to learn skills for employment & 30 \\
Having a serious health problem* & 29 \\
Not enough time for fun & 13 \\
No place to sleep & 12 \\
\hline
\end{tabular}

*Defined as a health problem that affects the respondent's ability to function in daily life somewhat or very much

Table 7 Psychosocial distress outcomes experienced sometimes or most of the time in the past month

\begin{tabular}{ll}
\hline & $\%$ \\
\hline Felt guilty & 76 \\
Felt worried or stressed & 70 \\
Felt irritated or bothered & 65 \\
Felt sad & 63 \\
Had headaches or stomachaches & 62 \\
Had trouble concentrating & 62 \\
Felt overwhelmed by their problems & 61 \\
Had nightmares & 57 \\
Felt hopeless about the future & 56 \\
Felt very tired & 52 \\
Felt nothing in life interested them & 51 \\
\hline
\end{tabular}

Several questions were posed to the subset of respondents who had lost a father, a mother, or both about their feelings of grief in relation to the death(s) they had experienced. Most (83 percent) reported that they still felt sad, 59 percent reported feelings of anger, and 41 percent reported fear. 


\section{Despite adverse circumstance, youth reported signs of psychosocial well-being and strength.}

Despite adverse circumstances and feelings of distress, there were signs of psychosocial well-being among youth (Table 8). Peer relationships emerged as an important source of social support and coping, as over 90 percent of youth enjoyed the time they spent with friends some or most of the time during the past month. The high proportion of youth who reported that they felt they could do things to help themselves, felt confident in themselves, felt hopeful about the future, and felt able to cope with difficulties in life suggests some level of resilience in this vulnerable group of youth. Another 90 percent of youth reported that they "make good choices" and 88 percent were resourceful enough to seek help from an adult if they have a problem.

Table 8 Psychosocial well-being outcomes experienced sometimes or most of the time in the past month

\begin{tabular}{ll}
\hline & $\%$ \\
\hline Enjoyed spending time with friends & 92 \\
Felt happy & 87 \\
Felt they can do things to help themselves & 79 \\
Felt confident in themselves & 79 \\
Felt hopeful about the future & 78 \\
Felt they can cope with difficulties & 69 \\
\hline
\end{tabular}

Thus despite hardships, many youth were able to maintain friendships, seek help from adults, feel confident and capable, enjoy and respect themselves, and maintain hope for the future. These manifestations of flexibility, learning, and adaptation are signs of resilience (Duncan and Arnston 2003) and positive coping - important aspects of psychosocial well-being.

There was also, however, a substantial minority of adolescents who lacked psychosocial well-being - who responded "never" to many of the items related to coping and resilience: 31 percent reported they were unable to cope with difficulties in their lives, 21 percent lack confidence in themselves, and 22 percent had no hope for the future.

\section{Exposure to stress, exposure to trauma, and social isolation were associated with psychosocial distress.}

Trauma scores, daily stress scores, and social support scores were each split into high, medium, and low categories as follows. Respondents were listed in the order of their scores and then divided into the three categories (high, medium, and low) with an equal number of respondents in each. These high, medium, 


\section{Hgrizons}

and low categories were then cross-tabulated with indicators of psychosocial well-being and distress. (See Appendix D, page 42, for the complete findings).

High trauma and high daily stress scores were both strongly associated with many depression- and anxiety-like symptoms characteristic of psychosocial distress. For example, respondents in the high trauma group compared to those in the low trauma group were much more likely to report feeling sad (74 percent vs. 53 percent), disinterested in life (61 percent vs. 39 percent), overwhelmed (74 percent vs. 47 percent), and hopeless (71 percent vs. 43 percent) sometimes or most of the time in the last month. Similarly, respondents in the high daily stress group compared to their peers in the low daily stress group were more likely to report feeling disinterested in life (57 percent vs. 41percent), overwhelmed (70 percent vs. 47 percent), and hopeless (68 percent vs. 43 percent) sometimes or most of the time in the last month.

High trauma and high daily stress scores were in general inversely associated with aspects of psychosocial well-being, although the differences were not as pronounced as they were for the psychosocial distress outcomes. For example, 67 percent of respondents in the low daily stress category reported "feeling like a valuable person," while only 55 percent of those in the high daily stress category reported feeling that way sometimes or most of the time in the last month.

High social support scores, on the other hand, were negatively associated with many psychosocial distress outcomes. For example, 45 percent of respondents with high social support scores reported feeling hopeless about the future sometimes or most of the time in the last month compared to 62 percent of those with low social support scores. High social support scores were positively associated with some aspects of psychosocial well-being (although the pattern was not as pronounced as it was for psychosocial distress). This supports the idea that a strong, supportive social network helps to mitigate psychosocial distress.

While a causal relationship cannot be inferred by these data, these associations suggest, as expected, a negative relationship between trauma and stress and the psychosocial well-being of youth, and a positive relationship between social support and emotional well-being and resilience. These findings help to support the measures chosen to assess psychosocial well-being and psychosocial distress.

One exception to the association between exposure to trauma and psychosocial distress was that respondents who reported the greatest exposure to trauma compared to those who reported the least exposure were more likely to be able to "cope with the difficulties in life” (73 percent vs. 63 percent). This suggests personal strength gained, rather than lost, as a result of moving through traumatic experiences. Post-traumatic growth, defined as positive psychological change or a greater sense of personal strength following trauma, has been discussed and studied in other contexts (Tedeschi and Calhoun 2004; Tedeschi and Calhoun 1996; and Linley and Joseph 2004). However, post-traumatic growth and psychosocial distress are not mutually exclusive. In fact they often co-exist (Cadell et al. 2003). Tedeschi and Calhoun (2004) point out "post-traumatic growth occurs in the context of suffering and significant psychological struggle.” 


\section{Relationships Between Psychosocial Outcomes and Respondents' Sex, Orphan Status, and Age}

\section{Although females reported greater social support, they experienced more stress, trauma, and psychosocial distress than males.}

Females as a group were younger and from poorer households than males. They also had greater exposure to deaths, stress in daily life, and traumatic experiences (Table 9). The inferior status of females in society and their traditional role as caregivers, which may cause them to be more directly in contact with illness, death, and AIDS-related stigma, may help to explain why females in this study were more vulnerable to stress, trauma, and psychosocial distress than males.

Table 9 Levels of trauma, daily stress, and social support by sex

\begin{tabular}{|c|c|c|}
\hline & $\begin{array}{c}\text { Males } \\
(n=604)\end{array}$ & $\begin{array}{l}\text { Females } \\
(n=654)\end{array}$ \\
\hline Mean trauma score ${ }^{1}$ (range $0-12$ ) & $5.3(0-12)$ & $5.8(0-12)$ \\
\hline Low trauma $(\%)^{2}$ & 39 & 30 \\
\hline Medium trauma $(\%)^{2}$ & 28 & 32 \\
\hline High trauma $(\%)^{2}$ & 33 & 38 \\
\hline Mean daily stress score ${ }^{1}$ (range $0-10$ ) & $4.4(1-10)$ & $4.7(0-10)$ \\
\hline Low daily stress (\%) & 35 & 30 \\
\hline Medium daily stress (\%) & 29 & 28 \\
\hline High daily stress (\%) & 36 & 42 \\
\hline Mean social support score ${ }^{1}$ (range $0-12$ ) & $7.7(3-12)$ & $8.2(3-12)$ \\
\hline Low social support (\%) ${ }^{2}$ & 49 & 36 \\
\hline Medium social support $(\%)^{2}$ & 32 & 35 \\
\hline High social support $(\%)^{2}$ & 19 & 29 \\
\hline
\end{tabular}

Given their higher levels of stress and trauma, it is not surprising that females in this study were more likely to report signs of psychosocial distress in the last month. For most of the 20 variables measuring psychosocial distress, a greater proportion of females reported negative feelings compared to males, and the differences were statistically significant for 10 of the variables (Table 10). For example, females were more likely to report physical manifestations of psychosocial distress, such as loss of appetite, headaches, and stomachaches. Although this may be partly due to greater emotional expressiveness among females, 


\section{Hgrizons}

the results strongly suggest that females experienced greater psychosocial distress, including depressionlike symptoms such as irritability, disinterest in life, feeling alone, somatic symptoms, and sadness.

Table 10 Sex differences in psychosocial distress and well-being outcomes experienced sometimes or most of the time in the last month

\begin{tabular}{lcc}
\hline & Males & Females \\
\hline Psychosocial distress & 60 & 69 \\
Felt irritable or bothered* & 43 & 54 \\
Felt nothing in life interested them* & 34 & 45 \\
Felt alone in the world* & 33 & 42 \\
Spent a lot of time sleeping* & 56 & 68 \\
Had headaches or stomachaches* & 56 & 68 \\
Had no appetite* & 38 & 53 \\
Felt sad* & 59 & 66 \\
Cried* & 44 & 64 \\
Had nightmares* & 59 \\
Felt others are targeting them & \\
Psychosocial well-being & 53 & 58 \\
Feel confident in themselves* & 50 & \\
Feel hopeful about the future* & & 74 \\
Enjoy spending time with friends* & 83 & 75 \\
\hline
\end{tabular}

${ }^{*} \mathrm{p}<.05$ (Chi-square)

\section{Orphaned youth report higher exposure to stress and lower levels of social support and psychosocial well-being than non-orphaned youth.}

All orphans (i.e. both double and single orphans) reported greater exposure to daily stress and perceived less social support than their non-orphaned peers (Table 11). Orphans were also more likely to report being rejected by family during times of need. Among all orphans not living with a surviving parent, 22 percent were made to feel unwelcome at home. Compared to non-orphans, orphans were far more likely to report that "not having an adult I can talk to" is a "big problem" (37 percent of non-orphans vs. 49 percent of orphans, $\mathrm{p}<0.05$ ). 
Table 11 Levels of daily stress and social support by orphan status

\begin{tabular}{|c|c|c|c|c|c|}
\hline & $\begin{array}{c}\text { Non- } \\
\text { orphans } \\
(n=436)\end{array}$ & $\begin{array}{c}\text { All } \\
\text { orphans } \\
(n=822)\end{array}$ & $\begin{array}{l}\text { Maternal } \\
\text { orphans } \\
(n=124)\end{array}$ & $\begin{array}{l}\text { Paternal } \\
\text { orphans } \\
(n=423)\end{array}$ & $\begin{array}{l}\text { Double } \\
\text { orphans } \\
(\mathrm{n}=275)\end{array}$ \\
\hline Mean daily stress scores $(0-10)$ & $4.1(0-9)$ & $4.8^{1}(0-10)$ & $4.7^{1}(0-9)$ & $5.0^{1}(0-10)$ & $4.5^{1}(0-10)$ \\
\hline Low daily stress (\%) & 40 & $28^{2}$ & 30 & $26^{2}$ & 32 \\
\hline Medium daily stress (\%) & 27 & $29^{2}$ & 32 & $28^{2}$ & 30 \\
\hline High daily stress (\%) & 34 & $43^{2}$ & 39 & $47^{2}$ & 39 \\
\hline Mean social support score (0-12) & $8.4(4-12)$ & $7.7^{1}(3-12)$ & $7.7^{1}(3-12)$ & $7.7^{1}(3-12)$ & $7.8^{1}(3-12)$ \\
\hline Low social support (\%) & 32 & $48^{2}$ & $46^{2}$ & $47^{2}$ & $49^{2}$ \\
\hline Medium social support (\%) & 34 & $33^{2}$ & $37^{2}$ & $36^{2}$ & $28^{2}$ \\
\hline High social support (\%) & 34 & $19^{2}$ & $17^{2}$ & $17^{2}$ & $24^{2}$ \\
\hline
\end{tabular}

${ }_{2}^{1} p<0.05$ (t-test comparing the mean score of non-orphans to the mean scores of each orphan group)

${ }^{2} p<0.05$ (Chi-Square comparing non-orphans to each orphan group)

Compared to non-orphans, single and/or double orphans were more likely to report signs of psychosocial distress in the past month for most of the 20 psychosocial distress outcomes. For six of these, the differences were statistically significant, and all were suggestive of depression: feeling overwhelmed, feeling alone in the world, having trouble concentrating, feeling worried or stressed, irritability, and loss of appetite (Table 12).

For two of the seven psychosocial well-being variables, some or all of the orphan groups reported significantly poorer outcomes than non-orphans (Table 12). Overall, orphans scored lower on all but one of the other psychosocial well-being variables, but the differences were not statistically significant.

Given that orphans in the sample were predominantly female (Table 13), we would expect them to have greater exposure to stress and more psychosocial distress. However, when we disaggregated the data by sex, both male and female orphans tended to have higher daily stress scores and lower social support scores than non-orphans (Appendix E, page 44) and higher levels of some psychosocial distress variables (Appendix F, page 45). 
Table 12 Psychosocial distress and well-being outcomes experienced sometimes or most of the time in the last month by orphan status (\%)

\begin{tabular}{lccccc}
\hline & $\begin{array}{c}\text { Non- } \\
\text { orphans } \\
(\mathbf{n = 4 3 6 )}\end{array}$ & $\begin{array}{c}\text { All } \\
\text { orphans } \\
(\mathbf{n = 8 2 2})\end{array}$ & $\begin{array}{c}\text { Maternal } \\
\text { orphans } \\
(\mathbf{n = 1 2 4})\end{array}$ & $\begin{array}{c}\text { Paternal } \\
\text { orphans } \\
(\mathbf{n = 4 2 3})\end{array}$ & $\begin{array}{c}\text { Double } \\
\text { orphans } \\
(\mathbf{n}=\mathbf{2 7 5})\end{array}$ \\
\hline Psychosocial distress & & & & & \\
Felt overwhelmed & 53 & $65^{1}$ & $69^{1}$ & $64^{1}$ & $64^{1}$ \\
Felt alone in the world & 36 & $42^{1}$ & $46^{1}$ & 41 & 41 \\
Had trouble concentrating & 56 & $65^{1}$ & $66^{1}$ & $65^{1}$ & 63 \\
Felt worried/stressed & 64 & $73^{1}$ & 73 & $75^{1}$ & 71 \\
Felt irritable & 58 & $68^{1}$ & 63 & $70^{1}$ & $68^{1}$ \\
Had no appetite & 42 & $48^{1}$ & 51 & $49^{1}$ & 45 \\
Psychosocial well-being & & & & & $57^{1}$ \\
Felt like a valuable person & 68 & $57^{1}$ & $54^{1}$ & $58^{1}$ & 77 \\
$\begin{array}{l}\text { Felt hopeful about the } \\
\text { future }\end{array}$ & 82 & $76^{1}$ & 76 & $75^{1}$ & \\
\hline
\end{tabular}

${ }^{1} p<0.05$ (Chi-Square comparing non-orphans to each orphan group)

Table 13 Sex distribution by orphan status (\%)

\begin{tabular}{lccccc}
\hline & $\begin{array}{c}\text { Non- } \\
\text { orphans } \\
(\mathbf{n}=\mathbf{4 3 6})\end{array}$ & $\begin{array}{c}\text { All } \\
\text { orphans* } \\
(\mathbf{n}=\mathbf{8 2 2})\end{array}$ & $\begin{array}{c}\text { Maternal } \\
\text { orphans* } \\
(\mathbf{n}=\mathbf{1 2 4})\end{array}$ & $\begin{array}{c}\text { Paternal } \\
\text { orphans } \\
(\mathbf{n}=\mathbf{4 2 3})\end{array}$ & $\begin{array}{c}\text { Double } \\
\text { orphans } \\
(\mathbf{n}=\mathbf{2 7 5})\end{array}$ \\
\hline Males & 53 & 45 & 39 & 48 & 45 \\
Females & 47 & 55 & 61 & 53 & 55 \\
\hline
\end{tabular}

${ }^{1} p<0.05$ (Chi-Square comparing non-orphans to each orphan group)

\section{Exposure to trauma and signs of psychosocial distress increased with age.}

Analysis by age was conducted using three age categories: 14 to 15 year olds, 16 to 18 year olds, and 19 to 20 year olds. These age groupings were chosen based on the school system, with 14 to 15 year olds expected to be in Forms 1 and 2, 16 to 18 year olds in Forms 3 and 4, and 19 to 20 year olds in high school Forms 5 and 6 or finished with school.

As shown in Table 14, older youth had a higher mean trauma score than younger youth, but the age groups were fairly similar with respect to daily stress and social support scores (see Appendix G, page 46, for separate results for males and females). 
Table 14 Levels of trauma, daily stress, and social support by age range

\begin{tabular}{|c|c|c|c|}
\hline & $\begin{array}{c}14-15 \\
\text { years old } \\
(n=590)\end{array}$ & $\begin{array}{c}16-18 \\
\text { years old } \\
(n=583)\end{array}$ & $\begin{array}{c}19-20 \\
\text { years old } \\
(n=85)\end{array}$ \\
\hline Mean trauma score $(0-12)$ & $5.4(0-12)$ & $5.6(0-12)$ & $6.4^{1}(0-12)$ \\
\hline Low trauma (\%) & 37 & 33 & $20^{2}$ \\
\hline Medium trauma (\%) & 31 & 29 & $32^{2}$ \\
\hline High trauma (\%) & 32 & 37 & $48^{2}$ \\
\hline Mean daily stress score $(0-10)$ & $4.5(0-10)$ & $4.6(0-10)$ & $4.7(0-9)$ \\
\hline Low daily stress (\%) & 34 & 31 & 27 \\
\hline Medium daily stress (\%) & 29 & 27 & 34 \\
\hline High daily stress (\%) & 37 & 42 & 39 \\
\hline Mean social support score (0-12) & $7.9(4-12)$ & $8.0(3-12)$ & $8.0(3-12)$ \\
\hline Low social support (\%) & 42 & 43 & 41 \\
\hline Medium social support (\%) & 34 & 33 & 33 \\
\hline High social support (\%) & 24 & 24 & 26 \\
\hline
\end{tabular}

The study findings also showed that reported psychosocial distress significantly increased with age for nine of the 19 variables studied (Table 15). The results may be partly explained by greater verbal ability of older youth in reporting their emotions and reflecting on their life circumstances. However, the findings were quite pronounced, suggesting increasing anxiety, hopelessness, and depression with age as young people confront even greater life challenges and accumulate stressful experiences. There were no significant differences across age groups with respect to manifestations of lingering grief (sadness, anger, or fear in relation to deaths) or psychosocial well-being.

\section{Despite greater exposure to traumatic events, older respondents reported greater confidence and self-esteem.}

Mean trauma scores were higher for older respondents than for younger ones (Table 14). Yet, 19- to 20year-old respondents were more likely to report feeling confident in themselves most of the time compared to 14 to 15 year olds (40 percent vs. 30 percent). Similarly, more than a fourth (27 percent) of 19 to 20 year olds reported feeling like a valuable person most or all of the time compared to only 18 percent of 14 to 15 year olds. This may reflect maturity and self-respect gained through enduring difficult circumstances, taking on responsibilities, and learning new coping and survival strategies. This also suggests growing resilience over time and resonates again with the concept of "post-traumatic growth," particularly in the form of improved self-perception and sense of personal strength. However, since the 


\section{Hgrizons}

majority of older respondents were enrolled in the study on the basis of having been peer leaders or mentors, this may also reflect a sense of self-worth, competency, and accomplishment gained from that experience as well as from normal maturation.

Table 15 Psychosocial distress experienced sometimes or most of the time in the last month by age range

\begin{tabular}{lccc}
\hline & $\begin{array}{c}\mathbf{1 4 - 1 5} \\
\text { years old }\end{array}$ & $\begin{array}{c}16-18 \\
\text { years old }\end{array}$ & $\begin{array}{c}\mathbf{1 9 - 2 0} \\
\text { years old }\end{array}$ \\
\hline $\begin{array}{l}\text { Psychosocial distress } \\
\text { Felt sad* }\end{array}$ & 57 & 68 & 68 \\
Had trouble concentrating* & 56 & 66 & 74 \\
Felt nothing in life interested them* & 44 & 53 & 54 \\
Felt alone in the world* & 35 & 43 & 48 \\
Felt overwhelmed by problems* & 55 & 65 & 71 \\
Couldn't control their emotions* & 41 & 49 & 51 \\
Felt hopeless about the future* & 51 & 59 & 66 \\
Felt worried or stressed* $^{*}$ & 64 & 74 & 81 \\
Felt irritable* $^{*}$ & 62 & 65 & 74 \\
\hline
\end{tabular}

${ }^{*} p<0.05$ (Chi-square)

\section{Associations Between Participation in Psychosocial Support I nterventions and Psychosocial Outcomes}

In exploring the effects of each program, the researchers focused on a limited number of key psychosocial outcomes clustered into three broad domains: psychosocial well-being, psychosocial distress, and lingering grief (Table 2, page 13 ). Appendix A (page 38) lists the exact wording of survey questions for each outcome and domain and Appendix C (page 40) describes the regression analysis process.

Regression analysis was used to control for confounding factors in the relationships between participation in psychosocial support interventions and outcomes. The final model contained seven covariates. These covariates were: age group (categorized into 14 to 15,16 to 18, and 19 to 20 year olds), province (Bulawayo, Midlands, North Matabeleland, and South Matabeleland), setting (urban, peri-urban, rural), possessions score, trauma score, daily stress score, and social support score (see Appendix B, page 39, for a description of the four scores). Rather than controlling for sex, regression was performed separately for males and females. Thus the full model was run separately for males and females against all of the outcomes of interest to produce the odds ratio estimates and confidence intervals found in the tables. This section presents the unadjusted proportions with selected psychosocial outcomes comparing each 
intervention group to the comparison group. Where a significant association in the multivariate analysis was found, the adjusted odds ratio is also presented.

Interestingly, two variables that were eliminated from the final model as a result of the analysis process were time since exposure to the death of a parent (within the last year or not) and orphan status (nonorphan, maternal, paternal, or double orphan). This was unexpected. However, the trauma score, which captured exposure to illness and death among both family and friends, remained in the model. Possible explanations include that the surviving, extended family helped to fill the role of the deceased parent and mitigate the trauma of loss. On the other hand, it is possible that since youth in the sample confronted an unusually high number of traumatic events and stressful conditions, the loss of a parent is one among many traumas and thus did not emerge as a singularly and exceptionally traumatic event. In this case the cumulative exposures to trauma and stress may be more important in influencing psychosocial well-being than whether or not a child has lost a parent per se. It is also possible that the researchers did not fully understand or capture youth's attitudes towards death, grieving processes, and signs and symptoms thereof, from a cultural perspective. In any case, orphaned youth reported more psychosocial distress than their non-orphaned peers as discussed earlier.

The data from the regression analysis is provided in its entirety in Appendix $\mathrm{H}$ (page 47). Results of the regression analysis are discussed separately for each intervention relative to the comparison group: youth participating in community PSS, youth participating in the Masiye Camp, and youth leaders. As part of the discussion of each intervention, males and females are discussed separately. Instances of adjusted odds ratios with a p-value of less than 0.05 are noted.

Due to the lack of a pre-post design and randomly selected study groups, no inferences can be drawn about causal relationships between the interventions and psychosocial outcomes (or the lack thereof). Associations are identified that may suggest, but do not prove, a causal relationship. This in turn paves the way for further research to test causal relationships.

\section{Community PSS was associated with greater confidence for males, but there were no other associations with psychosocial well-being for males or females.}

Males who participated in community PSS programs were significantly more likely than their comparison group peers to report self-confidence (85 percent vs. 77 percent, Adjusted OR: 1.85, CI: 1.03-3.33, $\mathrm{p}<0.05)$.

There were no other associations, either positive or negative, between participation in community PSS programs and other psychosocial well-being outcomes for either males or females, relative to the comparison group. Since the community-based PSS programs that were studied have only been providing services for a short period of time, they may be too weak, as yet, to have a quantifiable or enduring impact on psychosocial well-being. Further research is recommended once the community PSS programs are better established. 


\section{Hgrizons}

\section{Youth exposed to community PSS reported more signs of psychosocial distress and lingering grief.}

Males in the community PSS group were more likely than comparison group males to report several outcomes related to psychosocial distress, including feeling sad (64 percent vs. 58 percent), overwhelmed (64 percent vs. 53 percent), and not being interested in life (49 percent vs. 40 percent), although the differences were not statistically significant.

A significantly greater proportion of females exposed to community PSS reported sadness in the last month compared to the comparison group (77 percent vs. 58 percent, Adjusted OR: 2.18, CI: 1.39-3.43, $\mathrm{p}<0.05$.) and were also more likely to feel overwhelmed (64 percent vs. 57 percent), but this difference was not statistically significant.

Exposure to community PSS did not seem to be associated either positively or negatively with outcomes related to lingering grief for males. Females exposed to community PSS, however, were more likely to report lingering grief when thinking about their losses, especially in the form of anger (63 percent vs. 55 percent) and being scared (59 percent vs. 41 percent, Adjusted OR: 1.71, CI: 1.01-2.90, p < 0.05).

\section{The Salvation Army Masiye Camp experience was associated with greater self-confidence among males.}

Males who attended Masiye Camp were significantly more likely than comparison group males to report feeling confident in the last month (87 percent vs. 77 percent, Adjusted OR: 2.27, 1.14-4.45, p < 0.05). They were also more likely to feel that they could cope with difficulties in their lives (74 percent vs. 67 percent). Females who attended the Masiye Camp were also more likely than comparison group females to believe they could cope with difficulties in their lives (72 percent vs. 63 percent).

There were no other key associations between participation in the Masiye Camp and the other two psychosocial well-being outcomes (hopefulness and self-efficacy) for males or females. Nevertheless, the finding that Masiye Camp may be associated with greater self-confidence and coping, especially in males, is an important outcome that reflects the overall mission of the camp.

Although the differences were not statistically significant, the findings suggest greater psychosocial distress among youth who attended camp relative to comparison group youth. For example, camp group males were slightly more likely than comparison group males to report crying (50 percent vs. 43 percent), feeling overwhelmed (64 percent vs. 57 percent), feeling sad (58 percent vs. 55 percent), and feeling hopeless (65 percent vs. 54 percent). Similarly, females exposed to camp were more likely than comparison group females to report feeling overwhelmed (64 percent vs. 57 percent) and hopeless about the future (60 percent vs. 51 percent). They also were more likely than comparison group females to report lingering feelings of anger about the death of one or both parents (62 percent vs. 55 percent). 


\section{Leaders reported greater self-confidence and coping skills, but also more psychosocial distress than the comparison group.}

For males, the leadership experience was strongly associated with self-confidence (96 percent vs. 77 percent, Adjusted OR 6.83, CI: 1.49-31.34, $\mathrm{p}<0.05$ ). Male leaders were also more likely than comparison group males to report being able to cope with difficulties in life (84 percent vs. 67 percent).

Female leaders were more likely than comparison group females to report feeling confident (87 percent vs. 72 percent), able to do things to help themselves ( 84 percent vs. 76 percent), and feeling they could cope with difficulties (82 percent vs. 63 percent). Although none of the adjusted odds ratios were significant, this overall pattern may suggest that leadership fosters higher psychosocial well-being (especially confidence and self-efficacy) among females. Interestingly, among the interventions, the leadership experience was the one most strongly associated with psychosocial well-being outcomes for females.

Although a causal relationship cannot be proven, these findings suggest that youth who choose to be leaders or are chosen to be leaders may be more confident and able to cope to begin with, or that the experience of being a leader and mentor for other vulnerable youth may actually build confidence and coping skills. In fact, both statements may be true.

At the same time, male leaders were more likely than comparison group males to report several outcomes related to psychosocial distress, including feeling overwhelmed (73 percent vs. 53 percent) and feeling hopeless (64 percent vs. 54 percent). For females, reported psychosocial distress was greater for leaders compared to the comparison group for all outcomes; feeling sad (82 percent vs. 58 percent), crying (77 percent vs. 58 percent), feeling overwhelmed (79 percent vs. 57 percent), feeling hopeless about the future (63 percent vs. 51 percent), and feeling uninterested in life (70 percent vs. 54 percent). Although none of the adjusted odds ratios were significant for males or females, there is a pattern whereby leaders, particularly females, appear to experience greater psychosocial distress than their comparison group peers. 


\section{Hgrizons}

\section{Conclusions and Recommendations}

Most respondents had experienced multiple traumatic events, including the death of loved ones, illness in the family, stigma, and rejection in times of need. Respondents also identified numerous causes of stress in their daily lives, such as lack of money, job opportunities, and health care. The difficult political and economic context in modern-day Zimbabwe likely added to this. Another source of stress identified by youth was not having adults to talk to about relationships, problems, and other issues. This highlights the importance of adult guidance for youth.

Not surprisingly, indications of psychosocial distress were widespread. More than half of youth reported feelings of worry or stress, irritability, sadness, difficulty concentrating, being overwhelmed, and hopelessness. These are outcomes that, in a Western context, would be suggestive of depression and anxiety.

Social connectedness, such as having supportive relationships with primary caregivers and members of one's cultural or faith groups, is widely recognized as a protective factor that "buffers the consequences of negative experiences on children” (Duncan and Arnston 2003). Unfortunately, social support for vulnerable youth in this study was inconsistent. Answers to social support questions revealed a mixed picture, with about half of youth reporting feeling very well supported by adults in their lives and people in their religious community. However, a significant vulnerable group also emerged, who never felt supported by their community, peers, or people in their religious community. Of note were reports of abandonment by family and friends in times of need and of youth living with guardians who were made to feel unwelcome.

Despite cumulative stresses and trauma, responses also suggest resilience in the youth studied. Resilience is an individual's capacity to adapt, cope, and remain strong in the face of adversity, including stressful and traumatic events (Boyden et al. 2000). The majority of all youth in the sample reported confidence, capacity to help themselves, and hopefulness for the future. However, a very vulnerable group unable to cope with difficulties, lacking self-confidence, and pessimistic about the future also emerged.

In terms of vulnerability, the findings suggest that females, orphans, and older youth may be particularly disadvantaged. Females in this sample were more likely than males to have experienced the death of a loved one and other traumatic events and reported more sources of stress in their daily lives. They also showed higher levels of psychosocial distress, such as less hopefulness, trust in others, and selfconfidence, and were more likely to manifest this distress through somatic (physical) symptoms such as poor appetite and fatigue. The experience of orphanhood was associated with greater exposure to trauma and stress, less social support, and lower levels of psychosocial well-being. Compared to their peers, orphaned youth had poorer access to supportive adults in whom they can confide.

Older youth had higher trauma scores and reported more signs of psychosocial distress. At the same time, older respondents, many of whom were peer leaders, tended to report more self-confidence and selfesteem than younger respondents. 
When comparing the intervention groups to the comparison group, all three interventions were associated with greater self-confidence, especially among males. However, positive associations with the other three psychosocial well-being outcomes-hopefulness, self-efficacy, and ability to cope-were much more limited.

Why did youth who participated in the PSS interventions appear to have higher levels of psychosocial distress and lingering grief? It may be due to selection bias. That is, since communities select the most vulnerable youth to participate in the interventions, the intervention groups may have been more vulnerable to start with than the comparison group. Multivariate regression analysis should have controlled for this, but some differences may have still emerged as a result of unmeasured factors that were also correlated with the outcomes. It could be that the interventions encouraged youth to discuss their emotions and that as a result, participants became more emotionally expressive or more emotionally literate. But it is also possible that the programs brought out emotions without adequately helping youth to resolve them. Members of the leaders group may have been more emotionally literate because of the training they received in relation to emotional issues. And their elevated psychosocial distress could also reflect additional responsibilities they had taken on as mentors to younger children. Regardless of the explanation for higher psychosocial distress among counselors, the data suggest the need for thorough training and ongoing support and supervision to help leaders handle their own emotions as well as take on the emotional burden of caring for others.

A possible reason for greater psychosocial distress among youth exposed to Salvation Army Masiye Camp is that camp activities expose children to a very different world than the one they normally live in - a world that provides emotional and spiritual support, fun and leisure, and raises awareness of children's rights. When the camp is over, many children return to situations of poverty and difficulty, and sometimes abuse. Returning to the real world may be a "let down" and very upsetting. Their new awareness of their rights may not be welcomed by adults in their real world. And the youth may become frustrated by their inability to effect change in their situations. This may inadvertently contribute to psychosocial distress among the Masiye Camp youth. Again, careful follow-up in the community could help to sustain positive effects from the camp.

\section{Program I mplications}

\section{It is necessary to find creative ways of sensitizing parents and caregivers to children's needs to talk to someone about their feelings, relationships, and healthy life decisions.}

Youth in this study were very vocal about their need for guidance and support from adults, and for greater social support in general. High levels of social support were associated with greater self-esteem. An important role for PSS programs would be to link youth with trusted and reliable adults with whom they can discuss issues of importance to them. Community and peer support for vulnerable youth should also be encouraged and developed. 


\section{Hgrizons}

PSS programs need to be aware of the potential added vulnerability of females to trauma, daily stress, and psychosocial distress.

It may be necessary to strengthen the ability of programs to recognize and address some of the particular issues experienced by females, such as poor self-esteem. These programs should also learn to recognize physical complaints as possible indications of psychosocial distress. Some girls-only activities may be useful in addressing their particular needs and vulnerabilities.

\section{Older youth may benefit from tailored PSS programs that help them cope with increasing responsibilities.}

With age comes mounting pressure and stress, as well as increased exposure to trauma. To help cope with unemployment and relationship issues, PSS programs should develop ways to link older youth to adult mentors and social support services.

\section{PSS programs need to be responsive to the unique needs of orphaned adolescents without causing further stigmatization.}

In comparison to their non-orphaned peers, orphaned adolescents reported greater psychosocial distress. Programs must respond to the strains of parental illness and death among adolescents, but be cautious about targeting orphaned youth lest they further stigmatize them or neglect the needs of other vulnerable youth.

\section{PSS programs should explore better ways to address psychosocial distress on the one hand, while cultivating resilience on the other.}

Psychosocial distress was widespread among vulnerable youth in the study area. But youth were also drawing on their own constructive coping skills, and most demonstrated a degree of confidence in their ability to manage life stressors. Since psychosocial well-being and psychosocial distress are not mutually exclusive, programs must both promote psychosocial well-being and reduce psychosocial distress and grief. Programs should aim to ensure that the activities do not simply bring out emotions and encourage youth to express them in a supportive environment, but also help youth to cope with feelings, such as sadness, over time. PSS programs must be sensitive to each child's particular needs (such as children who require longer-term or more skilled support in grief resolution, protection from abuse, and so forth) and should incorporate ongoing, community-based follow-up. 


\section{PSS programs should be adapted to reflect children's grieving processes and attitudes toward illness, loss, and death in this specific cultural context.}

For example, grief counseling and its perceived emotional benefits to orphans and vulnerable children and youth is central to the Salvation Army Masiye Camp model but needs to be revisited. An approach that takes a longer-term perspective to the grieving process, and supports children appropriately at the various stages of that process may prove beneficial.

\section{A comprehensive response to the psychosocial needs of vulnerable youth must go beyond discrete PSS programs to address underlying contextual causes of trauma and stress.}

Adolescents in the study provinces confronted many traumatic experiences and high levels of day-to-day stress. This context represents a serious challenge for PSS programs. PSS can comfort, support, and guide youth, and can encourage confidence, self-esteem, and coping skills. However, it may not be realistic to expect the type of PSS programming assessed to vastly reduce psychosocial distress in the presence of multiple and pervasive causes of trauma and stress in this setting. Therefore, broader program and policy responses are needed.

\section{Research I mplications}

\section{The survey instrument needs to be tested for validity and reliability.}

Our attempts to measure psychosocial well-being and distress were supported by the expected associations found between stress and trauma, and psychosocial distress, and the associations between social support and psychosocial well-being. However the instrument should be further tested to ensure its validity and reliability in measuring psychosocial well-being, emotional distress, and grief among youth in Zimbabwe; its usefulness in other country contexts, cultures, and languages; and its ability to capture psychosocial issues relevant for youth of both sexes and different ages.

\section{Further research is needed to see what factors are associated with resilience and post- traumatic growth.}

Despite widespread trauma, daily stress, and psychosocial distress, many youth succeeded in maintaining self-confidence, hopefulness, and social connectedness.

\section{In-depth qualitative research is needed to better understand children's grieving processes.}

Such research would focus on cultural attitudes, and children's attitudes in particular, toward illness, loss, and death. 


\section{Hgrizons}

Quasi-experiments should be conducted to more rigorously assess the impact of PSS programs.

These would feature a pre-post design. Ideally, post-intervention observations would be conducted with youth participants soon after the intervention is completed and again after some time has lapsed to determine whether any impact on psychosocial well-being is sustained over time. Future research should also isolate what parts of interventions have what effects, and how certain components can be improved. For example, how can the adventure-based model used by Salvation Army Masiye Camp be adapted to more effectively promote self-esteem among girls? 


\section{References}

Boyden, Jo and Gillian Mann. 2000. “Children’s risk, resilience and coping in extreme situations,” Background paper to the Consultation on Children in Adversity, Oxford, September 9-12.

Cadell, S., C. Regehr, and D. Hemsworth. 2003. "Factors contributing to posttraumatic growth: a proposed structural equation model,” American Journal of Orthopsychiatry 73(3): 279-287.

Duncan, Joan and Laura Arnston. 2003. "Children in crisis: Good practices in evaluating psychosocial programming.” International Psychosocial Evaluation Committee and Save the Children, USA. Draft.

Kleinbaum, D.G. and M. Klein. 2002. Logistic Regression: A Self Learning Text, Second Edition.” New York: Springer-Verlag.

Linley, P.A. and S. Joseph. 2004. "Positive change following trauma and adversity: A review.” Journal of Traumatic Stress 17(1): 11-21.

Makame, V., C. Ani, S. Grantham-Mcgregor. 2002. "Psychological well-being of orphans in Dar es Salaam, Tanzania,” Acta Pediatrica 91:459-465.

Stein, Jo. 2003. "Sorrow makes children of us all: A literature review on the psychosocial impact of HIV/AIDS on children,” Centre for Social Science Research Working Paper No. 47. Cape Town: Centre for Social Science Research.

Tedeschi, Richard G. and Lawrence Calhoun. 1996. "The posttraumatic growth inventory: Measuring the positive legacy of trauma,” Journal of Traumatic Stress 9(3): 455-471.

—. 2004. "Posttraumatic growth: A new perspective on psychotraumatology," in Psychiatric Times 13(4).

Thurman, Tonya, Leslie Snider, and L. Nyirazinyoye. 2004. "The voice of child-headed households in Gikongoro, Rwanda: Psychosocial challenges and program suggestions. Summary of focus group findings,” paper presented at a data dissemination meeting in Rwanda.

UNAIDS. 2001. "Investing in our future: Psychosocial support for children affected by AIDS. A case study in Zimbabwe and the United Republic of Tanzania,” UNAIDS Best Practice Collection. Geneva: UNAIDS. 


\section{Hgrizons}

\section{APPENDIX A \\ Psychosocial Well-being and Psychosocial Distress I tems}

The following are words and phrases people use to describe how they feel. In the last month, please tell me if you have any of these feelings: (1) never/rarely, (2) sometimes, or (3) most of the time.

\section{Well-being}

Felt happy

Felt confident in yourself

Enjoyed spending time with friends

Felt you were a valuable person

Felt you could do things to help yourself

Felt hopeful about the future

Felt you could cope with difficulties in your life

$\begin{array}{lll}1 & 2 & 3 \\ 1 & 2 & 3 \\ 1 & 2 & 3 \\ 1 & 2 & 3 \\ 1 & 2 & 3 \\ 1 & 2 & 3 \\ 1 & 2 & 3\end{array}$

\section{Distress}

Felt sad

Had trouble concentrating

Felt nothing in life interested you

Felt badly about yourself

Felt alone in the world

Felt overwhelmed by your problems

Felt you can't control your emotions

Felt hopeless about your future

Spent a lot of time sleeping

Had headaches or stomachaches

Felt irritable or that many things bothered you

Had no appetite, not felt like eating meals

Felt very tired

Felt guilty for bad things that have happened in your life

Had sleeping problems

Had nightmares

Felt worried or stressed

Cried

Had trouble getting along with people

Felt like other people were targeting you or against you

$\begin{array}{lll}1 & 2 & 3 \\ 1 & 2 & 3 \\ 1 & 2 & 3 \\ 1 & 2 & 3 \\ 1 & 2 & 3 \\ 1 & 2 & 3 \\ 1 & 2 & 3 \\ 1 & 2 & 3 \\ 1 & 2 & 3 \\ 1 & 2 & 3 \\ 1 & 2 & 3 \\ 1 & 2 & 3 \\ 1 & 2 & 3 \\ 1 & 2 & 3 \\ 1 & 2 & 3 \\ 1 & 2 & 3 \\ 1 & 2 & 3 \\ 1 & 2 & 3 \\ 1 & 2 & 3 \\ 1 & 2 & 3\end{array}$

\section{Lingering grief about losses (asked only to those respondents who had experienced a death)}

Do you still feel sad when you think about your loss?

Do you still feel angry when you think about your loss?

Do you still feel scared when you think about your loss?

$\begin{array}{ll}\text { Yes } & \text { No } \\ \text { Yes } & \text { No } \\ \text { Yes } & \text { No }\end{array}$




\section{APPENDIX B Creation of the Composite Index Variables}

The trauma score is a cumulative measure of stressful and traumatic events that have occurred in the respondent's life. Answering yes to a question contributed one or two points to a respondent's overall score (range: 0 to 14). Local youth and psychologists were consulted to determine how to weight each trauma item. The items were the following: having been rejected, neglected, and/or abandoned by relatives/extended family when you needed them ( 2 points), having lost the support of friends when you needed them (1 point), either illness in your family or being in a family or household in which people are very sick (1 point), death of loved ones ( 2 points), having lost family lands or possessions ( 2 point), having left school when you did not want to (2 points), family strife/divorce etc. (2 points), and having experienced either sexual abuse or physical abuse (2 points). Respondents' scores were arranged sequentially and then divided into high, medium, and low categories to create a roughly equal number (one third) of respondents in each category. The result was the following: low trauma (a total score of 04), medium trauma (a total score of 5 or 6 ) and high trauma (a total score of 7-14).

The social support score is a cumulative measure of four items that ask about perceived social support from different groups: the adults in your life, peers, the general community, and your religious community. All questions were equally weighted. The response to each question contributed from one to three points to the overall score for a maximum score of 12: very well supported (3 points), sometimes supported ( 2 points), or almost never supported (1 point). The range of possible social support scores is 0 to 12. The scores were arranged sequentially and then divided into high, medium, and low categories with roughly an equal number (one third) of respondents in each category. The result was the following: low social support (a total score of $0-8$ ), medium social support (a total score of 8 or 9 ), and high social support (a total score of 10-12).

The daily stress score is a cumulative measure of perceived daily stressors. All of the following were equally weighted at 1 point each: having a health problem that affects daily life functioning, being the primary or next to primary caregiver for an ill person in the household, having a problem with either learning skills for employment or being able to find a job, having a problem finding a place to sleep, having a problem either having enough access to social and legal services or having access to health care, having enough money, having time/opportunity for fun, having information you need to make decisions in your life, having a problem feeling safe and secure in your every day life, and having a problem finding a job. The range of daily stress scores is 0 to 10 . The scores were arranged sequentially and then divided into high, medium, and low categories with roughly an equal number (one third) of respondents in each category. The result was the following: low daily stress (a total score of $0-3$ ), medium daily stress (a total score of 4 or 5), and high daily stress (a total score of 5-10).

The possessions score is a cumulative measure of whether a respondent possesses any of the following basic necessities: a blanket to sleep with, a spare set of clothes, and a pair of shoes (1 point each). The range of possible scores is 0 to 3 . 


\section{Hgrizons}

\section{APPENDIX C Regression Methods}

In exploring associations between program exposure and participants’ psychosocial well-being, researchers selected and focused on a limited number of key psychosocial outcomes, clustered into three broad domains (Table 2, page 13).

Psychosocial well-being and psychosocial distress questions related to emotions experienced in the past month had three answer categories: never or rarely, sometimes, or most of the time. For data analysis purposes, "sometimes" and "most of the time" responses were aggregated into one response category to create a bivariate variable with two response categories labeled "No" (never or rarely in the past month) and "Yes" (sometimes or most of the time in the past month). This was done because interviewers reported that respondents had difficulty with the distinction between "sometimes" and "most of the time." Collapsing these answer categories still allowed researchers to distinguish between the presence versus the absence of each emotion. Questions related to lingering grief were already in bivariate format (yes/no). (See Appendix A, page 38, for the exact wording of the questions in each domain.)

We analyzed quantitative data using SPSS for Windows, version 12.0.2. Separate frequencies of independent variables and outcomes were examined across study exposure groups, age categories, gender, and orphan status in order to begin the assessment for potential confounding, interaction, and effect modification. The cross-tabulations that exhibited a greater degree of association based on the Chi-square distribution were selected to be included in the model building process.

In order to narrow the number of outcomes for analysis, a process that was partly established prior to the study initiation was used. First, the outcomes were grouped into thematic blocks or domains as suggested in the proposal. Once the contents of these domains were established, questions that were reported as not being well understood by the respondents during the survey process were discarded. From the remaining list, a matrix of Pearson Correlation coefficients were calculated correlating every outcome against the other outcomes within each domain. These coefficients would serve two purposes: to further establish the cohesiveness of the domains and to assist in identifying outcomes within each domain that may replicate similar outcomes. Any outcomes that were highly correlated, $r=0.3$ or higher in most cases, were examined and the one found to be the most clear was included in the regression analyses to follow; the others were dropped from the analysis. This way, it was felt that the regression analysis would better cover a broader outcome universe without being repetitive.

With a list of quality outcomes established, the process of creating a multivariate model for each outcome against the dependent variable while controlling for independent covariates was initiated. Unconditional logistic regression was chosen as the best method to produce odds ratios and 95 percent confidence intervals from the data. It is a widely used mathematical modeling approach that can describe the relationship of exposure to an intervention with a dichotomous outcome while controlling for a number of independent covariates. Logistic regression is based on the logistic function, which ranges between 0 and 1. It models the probability that the exposure leads to the outcome, 1 being always associated while 0 deems no association. Furthermore, it allows for a variety of formats to be used when quantifying 
exposure and covariates such as ordinal, nominal, and continuous constructs as well as combinations of these variables to be included in a model.

All covariates determined to be of interest from the previous cross-tabulations were included in the initial model. A common SPSS option that is part of the logistic module called backward stepwise regression was selected to help identify covariates that did not need to be included in the model. This process, in a sense, sifts through all the covariates, retaining only those that would significantly change the model if removed. The backward stepwise regression algorithm uses a likelihood ratio test to compare each step of the model as it removes a covariate. This tests the null hypothesis that the coefficient of the covariate being removed from the model is equal to zero. If the ratio of the likelihood statistics between the model with the covariate and the model without the covariate is greater than a p-value of 0.05 under the Chisquare distribution, the test accepts the null hypothesis and the covariate is removed from the model. At this point the model continues to the next covariate or "step". In most cases, setting the inclusion value to be 0.05 or less could be considered stringent but we found this necessary in order to better focus on a select few covariates. It is statistical principle to always have a small and efficient ("parsimonious") model, because too many terms can make it impossible to understand the relationships between the covariates, the exposure, and the outcome. The process continues until only covariates that would significantly affect the model if removed remain.

The above process was performed once for each outcome, and a table of which covariates remained in each model was compiled. The result was the creation of a cumulative list of all covariates that were found significant in one, some, or all of the outcomes. Again this cumulative list of "significant" covariates was modeled against all outcomes but this time groups or chunks of covariates were removed. Again the likelihood ratio test was used to examine whether their inclusion or removal would significantly change the model's estimates following the null hypothesis that the coefficients of the variables selected to be included in the chunk are equal to zero. This process continued across all outcomes until one list of covariates was found to fit every outcome. The next step checked for interaction by age and sex with each study group, which were found for most outcomes. This prompted a decision to present the results by gender and control for age within each model. Our final model contained seven covariates. These covariates are age (categorized into 14 to 15,16 to 18, and 19 to 20 year olds), province (Bulawayo, Midlands, Matabeleland North, Matabeleland South), setting (urban, peri-urban, rural), possessions score, trauma score, daily stress score, and social support score. This full model was then run against all of the outcomes of interest to produce the odds ratio estimates and confidence intervals found in the tables. 


\section{Hgrizons}

Appendix D

Psychosocial outcomes experienced sometimes or most of the time in the last month by low, medium, and high trauma scores (\%)

\section{Psychosocial distress}

Felt sad*

Trouble concentrating*

Felt nothing in life interested them*

Felt alone in the world*

Felt overwhelmed by problems*

Felt they could not control emotions *

Felt hopeless about the future*

Felt worried or stressed*

Felt irritable*

Had no appetite

Felt very tired

Psychosocial well-being

Felt happy*

Felt confident in themselves

Enjoyed spending time with friends

Felt they were a valuable person*

Felt they could do things to help themselves

Felt hopeful about the future*

Felt they could cope with difficulties in their life*

$\begin{array}{ccc}\text { Low } & \text { Medium } & \text { High } \\ & & \\ 53 & 61 & 74 \\ 49 & 62 & 74 \\ 39 & 46 & 61 \\ 26 & 43 & 50 \\ 47 & 60 & 74 \\ 35 & 45 & 56 \\ 43 & 52 & 71 \\ 57 & 72 & 81 \\ 52 & 63 & 78 \\ 36 & 46 & 55 \\ 45 & 52 & 59 \\ & & \\ 89 & 90 & 82 \\ 77 & 79 & 80 \\ 93 & 90 & 92 \\ 64 & 63 & 55 \\ 79 & 79 & 78 \\ 81 & 80 & 74 \\ 63 & 70 & 73\end{array}$

${ }^{*} \mathrm{p}<0.05$ (Chi-square)

Psychosocial outcomes experienced sometimes or most of the time in the last month by low, medium, and high daily stress scores (\%)

Psychosocial distress

Felt sad*

Trouble concentrating*

Felt nothing in life interests them*

Felt alone in the world ${ }^{*}$

Felt overwhelmed by problems*

Felt they could not control emotions*

Felt hopeless about the future*

Felt worried or stressed*

Felt irritable*

Had no appetite

Felt very tired

Psychosocial well-being

Felt happy*

Felt confident in themselves*

Enjoyed spending time with friends

Felt they were a valuable person*

Felt they could do things to help themselves*

Felt hopeful about the future*

Felt they could cope with difficulties in their life

$\begin{array}{ccc}\text { Low } & \text { Medium } & \text { High } \\ 63 & 59 & 66 \\ 51 & 63 & 69 \\ 41 & 47 & 57 \\ 30 & 39 & 49 \\ 47 & 62 & 70 \\ 39 & 41 & 54 \\ 43 & 54 & 68 \\ 57 & 72 & 81 \\ 55 & 66 & 72 \\ 41 & 40 & 53 \\ 53 & 47 & 55 \\ & & \\ 92 & 84 & 84 \\ 82 & 81 & 75 \\ 93 & 90 & 92 \\ 67 & 54 & 55 \\ 84 & 78 & 75 \\ 87 & 79 & 70 \\ 71 & 67 & 68\end{array}$

${ }^{*} p<0.05$ (Chi-square) 
Psychosocial outcomes experienced sometimes or most of the time in the last month by low, medium, and high social support scores (\%)

\section{Psychosocial distress}

Felt sad

Had trouble concentrating

Felt nothing in life interests them

Felt alone in the world

Felt overwhelmed by problems*

Felt they could not control emotions

Felt hopeless about the future*

Felt worried or stressed*

Felt irritable or that things bothered them*

Had no appetite

Felt very tired

$\begin{array}{ccc}\text { Low } & \text { Medium } & \text { High } \\ 63 & 65 & 58 \\ 63 & 64 & 57 \\ 50 & 51 & 43 \\ 40 & 42 & 37 \\ 67 & 60 & 51 \\ 45 & 48 & 42 \\ 62 & 56 & 45 \\ 74 & 72 & 59 \\ 68 & 65 & 58 \\ 46 & 47 & 42 \\ 50 & 54 & 53 \\ & & \\ 84 & 89 & 89 \\ 76 & 80 & 81 \\ 90 & 93 & 94 \\ 51 & 66 & 71 \\ 76 & 77 & 86 \\ 71 & 82 & 85 \\ 67 & 73 & 67\end{array}$

Psychosocial well-being

Felt happy*

Felt confident in themselves

Enjoyed spending time with friends

Felt they were a valuable person*

Felt they could do things to help themselves

Felt hopeful about the future*

Felt they could cope with difficulties in their life

67

73

67

${ }^{*} p<0.05$ (Chi-square) 


\section{APPENDIXE \\ Levels of Daily Stress and Social Support by Orphan Status, Stratified by Sex}

Males

\begin{tabular}{|c|c|c|c|c|c|}
\hline & $\begin{array}{c}\text { Non- } \\
\text { orphans } \\
(n=230)\end{array}$ & $\begin{array}{c}\text { All } \\
\text { orphans } \\
(n=374)\end{array}$ & $\begin{array}{c}\text { Maternal } \\
\text { orphans } \\
(n=48)\end{array}$ & $\begin{array}{l}\text { Paternal } \\
\text { orphans } \\
(n=201)\end{array}$ & $\begin{array}{l}\text { Double } \\
\text { orphans } \\
(n=125)\end{array}$ \\
\hline Mean daily stress score $(0-10)$ & $3.9(0-9)$ & $4.7^{1}(0-10)$ & $4.7^{1}(1-8)$ & $4.9^{1}(0-9)$ & $4.4^{1}(0-10)$ \\
\hline Low daily stress (\%) & 44 & $29^{2}$ & $27^{2}$ & $27^{2}$ & 34 \\
\hline Medium daily stress (\%) & 27 & $30^{2}$ & $42^{2}$ & $28^{2}$ & 29 \\
\hline High daily stress (\%) & 30 & $41^{2}$ & $31^{2}$ & $45^{2}$ & 37 \\
\hline Mean social support score (0-12) & $8.1(4-12)$ & $7.5^{1}(3-12)$ & $7.4^{1}(4-11)$ & $7.4^{1}(3-12)$ & $7.7(3-12)$ \\
\hline Low social support (\%) & 40 & $55^{2}$ & 52 & $57^{2}$ & $54^{2}$ \\
\hline Medium social support (\%) & 35 & $30^{2}$ & 35 & $32^{2}$ & $25^{2}$ \\
\hline High social support (\%) & 25 & $15^{2}$ & 13 & $11^{2}$ & $22^{2}$ \\
\hline
\end{tabular}

${ }_{1}^{1} p<0.05$ (t-test comparing the mean score of non-orphans to the mean scores of each orphan group)

${ }^{2} p<0.05$ (Chi-Square test comparing non-orphans to each orphan group)

\section{Females}

\begin{tabular}{|c|c|c|c|c|c|}
\hline & $\begin{array}{c}\text { Non- } \\
\text { orphans } \\
(n=206)\end{array}$ & $\begin{array}{c}\text { All } \\
\text { orphans } \\
(n=448)\end{array}$ & $\begin{array}{l}\text { Maternal } \\
\text { orphans } \\
(n=76)\end{array}$ & $\begin{array}{l}\text { Paternal } \\
\text { orphans } \\
(n=222)\end{array}$ & $\begin{array}{l}\text { Double } \\
\text { orphans } \\
(n=150)\end{array}$ \\
\hline Mean daily stress score $(0-10)$ & $4.4(0-9)$ & $4.9^{1}(0-10)$ & $4.7(0-9)$ & $5.1^{1}(0-10)$ & $4.6(0-10)$ \\
\hline Low daily stress (\%) & 35 & 28 & 32 & $25^{2}$ & 29 \\
\hline Medium daily stress (\%) & 27 & 28 & 25 & $28^{2}$ & 31 \\
\hline High daily stress (\%) & 38 & 44 & 43 & $48^{2}$ & 40 \\
\hline Mean social support score $(0-12)$ & $8.8(4-12)$ & $7.9^{1}(3-12)$ & $7.4^{1}(4-11)$ & $7.9^{1}(4-12)$ & $7.8^{1}(3-12)$ \\
\hline Low social support (\%) & 23 & $41^{2}$ & $42^{2}$ & $39^{2}$ & $45^{2}$ \\
\hline Medium social support (\%) & 34 & $36^{2}$ & $38^{2}$ & $39^{2}$ & $30^{2}$ \\
\hline High social support (\%) & 43 & $23^{2}$ & $20^{2}$ & $22^{2}$ & $25^{2}$ \\
\hline
\end{tabular}

${ }^{1} p<0.05$ (t-test comparing the mean score of non-orphans to the mean scores of each orphan group)

${ }^{2} p<0.05$ (Chi-Square test comparing non-orphans to each orphan group) 


\section{APPENDIX F \\ Psychosocial Well-being and Distress Outcomes by Orphans Status, Stratified by Sex}

Males

\begin{tabular}{|c|c|c|c|c|c|}
\hline & $\begin{array}{c}\text { Non- } \\
\text { orphans } \\
(\mathrm{n}=230)\end{array}$ & $\begin{array}{c}\text { All } \\
\text { orphans } \\
(\mathrm{n}=\mathbf{3 7 4})\end{array}$ & $\begin{array}{c}\text { Maternal } \\
\text { orphans } \\
(n=48)\end{array}$ & $\begin{array}{l}\text { Paternal } \\
\text { orphans } \\
(n=201)\end{array}$ & $\begin{array}{c}\text { Double } \\
\text { orphans } \\
(n=125) \\
\end{array}$ \\
\hline \multicolumn{6}{|l|}{ Psychosocial distress } \\
\hline Felt overwhelmed & 50 & $64^{1}$ & $73^{1}$ & $64^{1}$ & 61 \\
\hline Felt alone in the world & 30 & 37 & $46^{1}$ & 36 & 34 \\
\hline Had trouble concentrating & 57 & 61 & 65 & 63 & 55 \\
\hline Felt worried/stressed & 62 & $72^{1}$ & 69 & $73^{1}$ & 71 \\
\hline Felt irritable & 53 & $65^{1}$ & 56 & $68^{1}$ & 63 \\
\hline Felt hopeless about the future & 54 & 60 & $71^{1}$ & 60 & 57 \\
\hline Had no appetite & 31 & $42^{1}$ & 35 & $47^{1}$ & 35 \\
\hline Felt very tired & 52 & 49 & 58 & 48 & 47 \\
\hline \multicolumn{6}{|l|}{ Psychosocial well-being } \\
\hline Felt like a valuable person & 69 & $59^{1}$ & 56 & $57^{1}$ & 63 \\
\hline Felt hopeful about the future & 85 & $78^{1}$ & 79 & $78^{1}$ & 79 \\
\hline \multicolumn{6}{|c|}{${ }^{1} p<0.05$ (Chi-square comparing non-orphans to each orphan group) } \\
\hline \multicolumn{6}{|l|}{ Females } \\
\hline & $\begin{array}{c}\text { Non- } \\
\text { orphans } \\
(n=206)\end{array}$ & $\begin{array}{c}\text { All } \\
\text { orphans } \\
(n=448)\end{array}$ & $\begin{array}{c}\text { Maternal } \\
\text { orphans } \\
(n=76)\end{array}$ & $\begin{array}{l}\text { Paternal } \\
\text { orphans } \\
(\mathrm{n}=222)\end{array}$ & $\begin{array}{l}\text { Double } \\
\text { orphans } \\
(n=150)\end{array}$ \\
\hline \multicolumn{6}{|l|}{ Psychosocial distress } \\
\hline Felt overwhelmed & 56 & $65^{1}$ & 66 & 64 & 66 \\
\hline Felt alone in the world & 41 & 46 & 46 & 46 & 47 \\
\hline Had trouble concentrating & 55 & $68^{1}$ & 67 & $67^{1}$ & $70^{1}$ \\
\hline Felt worried/stressed & 66 & $74^{1}$ & 75 & $77^{1}$ & 70 \\
\hline Felt irritable & 64 & 71 & 67 & 71 & 73 \\
\hline Felt hopeless about the future & 50 & 56 & 54 & 55 & 58 \\
\hline Had no appetite & 53 & 53 & 61 & 50 & 53 \\
\hline Felt very tired & 54 & 54 & 62 & 52 & 53 \\
\hline \multicolumn{6}{|l|}{ Psychosocial well-being } \\
\hline Felt like a valuable person & 66 & $55^{1}$ & $53^{1}$ & 58 & $52^{1}$ \\
\hline Felt hopeful about the future & 79 & 74 & 74 & 73 & 75 \\
\hline
\end{tabular}

${ }^{1} p<0.05$ (Chi-square comparing non-orphans to each orphan group) 


\section{APPENDIX G \\ Levels of Trauma, Daily Stress, and Social Support by Age Group, Disaggregated by Sex}

\begin{tabular}{|c|c|c|c|}
\hline & $\begin{array}{c}14-15 \text { years old } \\
(n=246)\end{array}$ & $\begin{array}{c}16-18 \text { years old } \\
(n=309)\end{array}$ & $\begin{array}{c}19-20 \text { years old } \\
(n=49)\end{array}$ \\
\hline Mean trauma score (0-12) & $5.0(0-12)$ & $5.3(0-12)$ & $6.4^{1}(1-12)$ \\
\hline Low trauma $(\%)$ & 43 & 38 & $20^{2}$ \\
\hline Medium trauma (\%) & 29 & 28 & $31^{2}$ \\
\hline High trauma $(\%)$ & 29 & 34 & $49^{2}$ \\
\hline Mean daily stress score $(0-10)$ & $4.3(0-10)$ & $4.4(0-9)$ & $4.7(0-8)$ \\
\hline Low daily stress (\%) & 39 & 33 & 29 \\
\hline Medium daily stress (\%) & 29 & 28 & 34 \\
\hline High daily stress (\%) & 32 & 40 & 37 \\
\hline Mean social support score $(0-12)$ & $7.7(3-12)$ & $7.7(3-12)$ & $7.6(3-12)$ \\
\hline Low social support (\%) & 51 & 48 & 51 \\
\hline Medium social support (\%) & 30 & 34 & 31 \\
\hline High social support (\%) & 20 & 18 & 18 \\
\hline
\end{tabular}

${ }_{1}^{1} p<0.05$ (t-test comparing the mean score of $14-15$ year olds to the mean score of the other age groups)

${ }^{2} \mathrm{p}<0.05$ (Chi-square comparing $14-15$ year olds to the other age groups)

\begin{tabular}{|c|c|c|c|}
\hline & $\begin{array}{l}14-15 \text { years old } \\
(n=344)\end{array}$ & $\begin{array}{c}16-18 \text { years old } \\
(n=274)\end{array}$ & $\begin{array}{c}19-20 \text { years old } \\
(n=36)\end{array}$ \\
\hline Mean trauma score (0-12) & $5.5(0-12)$ & $6.0^{1}(0-12)$ & $6.4(1-11)$ \\
\hline Low trauma $(\%)$ & 33 & 28 & 19 \\
\hline Medium trauma (\%) & 32 & 31 & 33 \\
\hline High trauma (\%) & 35 & 41 & 47 \\
\hline Mean daily stress score $(0-10)$ & $4.6(0-9)$ & $4.8(0-10)$ & $4.8(0-9)$ \\
\hline Low daily stress (\%) & 31 & 28 & 25 \\
\hline Medium daily stress (\%) & 29 & 26 & 33 \\
\hline High daily stress (\%) & 40 & 45 & 42 \\
\hline Mean social support score $(0-12)$ & $8.1(3-12)$ & $8.2(4-12)$ & $8.5(5-11)$ \\
\hline Low social support (\%) & 35 & 38 & 28 \\
\hline Medium social support (\%) & 37 & 33 & 36 \\
\hline High social support (\%) & 28 & 29 & 36 \\
\hline
\end{tabular}

${ }^{1} p<0.05$ (t-test comparing the mean score of $14-15$ year olds to the mean score of the other age groups)

${ }^{2} \mathrm{p}<0.05$ (Chi-square comparing $14-15$ year olds to the other age groups) 


\section{APPENDI X H \\ Complete Regression Results, Stratified by Sex}

\begin{tabular}{|c|c|c|c|c|c|c|c|c|}
\hline \multicolumn{9}{|c|}{ Felt confident in themselves some or most of the time in the past month } \\
\hline \multicolumn{3}{|l|}{ Females } & \multicolumn{3}{|c|}{ Unadjusted } & \multicolumn{3}{|c|}{ Full model } \\
\hline Exposure & $\mathbf{n}$ & $\%$ & OR & $95 \% \mathrm{Cl}$ & p-value & OR & $95 \% \mathrm{Cl}$ & p-value \\
\hline Comparison & 290 & 72.4 & 1.00 & & & 1.00 & & \\
\hline Camp only & 115 & 71.3 & 0.95 & $0.59-1.53$ & 0.822 & 0.69 & $0.39-1.23$ & 0.210 \\
\hline Leader & 56 & 87.5 & 2.67 & $1.16-6.13$ & $0.021^{1}$ & 1.74 & $0.67-4.53$ & 0.259 \\
\hline Community PSS & 191 & 75.9 & 1.20 & $0.79-1.83$ & 0.393 & 1.10 & $0.69-1.76$ & 0.679 \\
\hline Total & 652 & & & & & & & \\
\hline \multicolumn{9}{|l|}{ Males } \\
\hline \multicolumn{9}{|l|}{ Exposure } \\
\hline Comparison & 267 & 77.5 & 1.00 & & & 1.00 & & \\
\hline Camp only & 133 & 87.2 & 1.98 & $1.10-3.55$ & $0.022^{1}$ & 2.27 & $1.14-4.45$ & $0.017^{1}$ \\
\hline Leader & 55 & 96.4 & 7.68 & $1.82-32.45$ & $0.006^{1}$ & 6.83 & $1.49-31.34$ & $0.014^{1}$ \\
\hline Community PSS & 149 & 85.2 & 1.67 & $0.98-2.86$ & $0.060^{2}$ & 1.85 & $1.03-3.33$ & $0.039^{1}$ \\
\hline Total & 604 & & & & & & & \\
\hline
\end{tabular}

Felt hopeful about the future some or most of the time in the past month

Females

Exposure

Comparison

Camp only

Leader

Community PSS

Total

Males

Exposure

Comparison

Camp only

Leader

Community PSS

Total

${ }_{1} p<0.05$
$2 p<0.10$
Full model

\begin{tabular}{ccc|ccc} 
OR & 95\% Cl & p-value & $\begin{array}{c}\text { OR } \\
1.00\end{array}$ & $95 \%$ Cl & p-value \\
1.00 & & & & \\
0.95 & $0.58-1.54$ & 0.821 & 0.78 & $0.43-1.41$ & 0.411 \\
1.46 & $0.72-2.96$ & 0.296 & 1.31 & $0.55-3.11$ & 0.537 \\
1.17 & $0.76-1.79$ & 0.482 & 0.97 & $0.60-1.57$ & 0.907 \\
& & & & & \\
& & & & & \\
1.00 & & & & & \\
1.10 & $0.65-1.86$ & 0.735 & 1.27 & $0.68-2.38$ & 0.447 \\
1.01 & $0.49-2.09$ & 0.970 & 0.87 & $0.37-2.15$ & 0.789 \\
1.26 & $0.75-2.12$ & 0.391 & 1.28 & $0.72-2.29$ & 0.402 \\
& & & & &
\end{tabular}




\section{Hgrizons}

Psychosocial well-being outcomes by study group, stratified by sex (continued) Felt they could do things to help themselves some or most of the time in the past month

Females

Exposure

Comparison

Camp only

Leader

Community PSS

Total

Males

Exposure

Comparison

Camp only

Leader

Community PSS

Total
Unadjusted

95\% Cl

OR

1.00

1.39

115

57

190

649

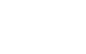

\begin{tabular}{l|l}
77.4 & 1.06
\end{tabular}

0.69-1.64

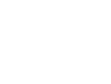

\section{Felt they}

Exposure

Comparison

Camp only

Leader

Community PSS

Total

Males

Exposure

Comparison

Camp only

Leader

Community PSS

Total

Full model

95\% Cl p-value

1.00

$1.68 \quad 0.89-3.15 \quad 0.109$

$1.96 \quad 0.79-4.84 \quad 0.145$

$0.99 \quad 0.61-1.61 \quad 0.974$

0.788

79.3
78.9
85.5
77.9

1.00

0.98

$0.98 \quad 0.59-1.63$

0.931

1.00

$1.13 \quad 0.63-2.03 \quad 0.684$

$1.53 \quad 0.68-3.43 \quad 0.300$

1.60

0.64-4.00

0.316

0.92

0.56-1.49

0.725

0.97

0.57-1.64

0.896

603

in the past month 
Psychosocial distress outcomes by study group, stratified by sex Felt sad some or most of the time in the past month

Females

Exposure

Comparison

Camp only

Leader

Community PSS

Total

Males

Exposure

Comparison

Camp only

Leader

Community PSS

Total

\begin{tabular}{cc|}
$\mathbf{N}$ & $\%$ \\
290 & 57.6 \\
115 & 60.9 \\
57 & 82.5 \\
191 & 77.0 \\
653 &
\end{tabular}

Unadjusted

OR $\quad 95 \% \mathrm{Cl} \quad$ p-value

1.00

1.15

3.46

2.46

$1.68-7.12$

$1.63-3.71$

$0.001^{1}$

$0.000^{1}$

1.631

Full model

95\% Cl p-value

1.00

$0.74 \quad 0.43-1.27 \quad 0.272$

$1.65 \quad 0.71-3.87 \quad 0.247$

2.18

$1.39-3.43$

$0.001^{1}$

\section{Cried some or most of the time in the past month}

Females

Exposure

Comparison

Camp only

Leader

Community PSS

Total

Males

Exposure

Comparison

Camp only

Leader

Community PSS

Total

\begin{tabular}{|c|c|c|c|c|c|c|c|}
\hline \multirow[b]{2}{*}{$\mathbf{N}$} & \multirow[b]{2}{*}{$\%$} & \multicolumn{3}{|c|}{ Unadjusted } & \multicolumn{3}{|c|}{ Full model } \\
\hline & & OR & $95 \% \mathrm{Cl}$ & p-value & OR & $95 \% \mathrm{Cl}$ & $p$-value \\
\hline 288 & 61.5 & 1.00 & & & 1.00 & & \\
\hline 115 & 60.9 & 0.98 & $0.63-1.52$ & 0.913 & 0.90 & $0.53-1.52$ & 0.684 \\
\hline 57 & 78.9 & 2.35 & $1.19-4.64$ & $0.014^{1}$ & 1.90 & $0.85-4.23$ & 0.117 \\
\hline 190 & 65.3 & 1.18 & $0.81-1.73$ & 0.399 & 1.30 & $0.85-2.00$ & 0.230 \\
\hline 650 & & & & & & & \\
\hline 267 & 43.4 & 1.00 & & & 1.00 & & \\
\hline 131 & 50.4 & 1.32 & $0.87-2.01$ & 0.192 & 0.98 & $0.60-1.62$ & 0.948 \\
\hline 55 & 40.0 & 0.87 & $0.48-1.57$ & 0.638 & 0.61 & $0.30-1.24$ & 0.168 \\
\hline 149 & 40.9 & 0.90 & $0.60-1.36$ & 0.620 & 0.80 & $0.51-1.26$ & 0.339 \\
\hline 602 & & & & & & & \\
\hline
\end{tabular}

$1 p<0.05$
$2 p<0.10$ 


\section{Hgrizons}

Psychosocial distress outcomes by study group, stratified by sex (continued) Felt overwhelmed by their problems some or most of the time in the past month

Females

Exposure

Comparison

Camp only

Leader

Community PSS

Total

Exposure

Comparison

Camp only

Leader

Community PSS

Total

\begin{tabular}{rc|ccl|} 
& & \multicolumn{3}{|c|}{ Unadjusted } \\
$\mathbf{n}$ & $\%$ & OR & $\mathbf{9 5 \%} \mathbf{C l}$ & p-value \\
290 & 56.9 & 1.00 & & \\
115 & 64.3 & 1.37 & $0.88-2.14$ & 0.170 \\
57 & 78.9 & 2.84 & $1.44-560$ & $\mathbf{0 . 0 0 3}^{\mathbf{1}}$ \\
191 & 63.9 & 1.34 & $0.92-1.95$ & 0.127
\end{tabular}

Full model

$95 \% \mathrm{Cl} \quad$-value

1.00

$0.98 \quad 0.57-1.69 \quad 0.938$

$\begin{array}{lll}1.79 & 0.79-4.04 & 0.162\end{array}$

$1.11 \quad 0.72-1.70 \quad 0.643$

Felt hopeless about the future some or most of the time in the past month

\begin{tabular}{|c|c|c|c|c|c|c|c|c|}
\hline \multicolumn{3}{|l|}{ Females } & \multicolumn{3}{|c|}{ Unadjusted } & \multicolumn{3}{|c|}{ Full model } \\
\hline Exposure & N & $\%$ & OR & $95 \% \mathrm{Cl}$ & $p$-value & OR & $95 \% \mathrm{Cl}$ & $p$-value \\
\hline Comparison & 290 & 50.7 & 1.00 & & & 1.00 & & \\
\hline Camp only & 115 & 60.0 & 1.46 & $0.94-2.26$ & $0.091^{2}$ & 1.11 & $0.65-1.89$ & 0.698 \\
\hline Leader & 57 & 63.2 & 1.67 & $0.93-2.99$ & $0.087^{2}$ & 1.00 & $0.48-2.10$ & 0.987 \\
\hline Community PSS & 191 & 51.8 & 1.05 & $0.73-1.51$ & 0.806 & 1.12 & $0.73-1.42$ & 0.709 \\
\hline Total & 653 & & & & & & & \\
\hline \multicolumn{9}{|l|}{ Males } \\
\hline \multicolumn{9}{|l|}{ Exposure } \\
\hline Comparison & 266 & 53.8 & 1.00 & & & 1.00 & & \\
\hline Camp only & 133 & 65.4 & 1.63 & $1.06-2.50$ & $0.027^{1}$ & 1.06 & $0.63-1.78$ & 0.833 \\
\hline Leader & 55 & 63.6 & 1.51 & $0.83-2.74$ & 0.182 & 0.89 & $0.42-1.89$ & 0.759 \\
\hline Community PSS & 149 & 57.0 & 1.14 & $0.76-1.71$ & 0.519 & 1.06 & $0.67-1.68$ & 0.802 \\
\hline Total & 603 & & & & & & & \\
\hline
\end{tabular}

${ }_{2}^{1} \mathrm{p}<0.05$

${ }^{2} p<0.10$ 
Psychosocial distress outcomes by study group, stratified by sex (continued) Felt nothing in life interested them some or most of the time in the past month

\begin{tabular}{|c|c|c|c|c|c|c|c|c|}
\hline \multicolumn{3}{|l|}{ Females } & \multicolumn{3}{|c|}{ Unadjusted } & \multicolumn{3}{|c|}{ Full model } \\
\hline Exposure & $\mathbf{n}$ & $\%$ & OR & $95 \% \mathrm{Cl}$ & p-value & OR & $95 \% \mathrm{Cl}$ & p-value \\
\hline Comparison & 288 & 54.2 & 1.00 & & & 1.00 & & \\
\hline Camp only & 115 & 49.6 & 0.83 & $0.54-1.28$ & 0.404 & 0.71 & $0.42-1.20$ & 0.199 \\
\hline Leader & 57 & 70.2 & 1.99 & $1.08-3.68$ & $0.028^{1}$ & 1.50 & $0.71-3.14$ & 0.287 \\
\hline Community PSS & 191 & 52.9 & 0.95 & $0.66-1.37$ & 0.782 & 0.87 & $0.57-1.31$ & 0.498 \\
\hline Total & 651 & & & & & & & \\
\hline \multicolumn{9}{|l|}{ Males } \\
\hline \multicolumn{9}{|l|}{ Exposure } \\
\hline Comparison & 267 & 40.4 & 1.00 & & & 1.00 & & \\
\hline Camp only & 133 & 41.4 & 1.04 & $0.68-1.59$ & 0.862 & 0.84 & $0.51-1.38$ & 0.490 \\
\hline Leader & 55 & 41.8 & 1.06 & $0.59-1.91$ & 0.851 & 0.81 & $0.40-1.65$ & 0.568 \\
\hline Community PSS & 149 & 49.0 & 1.41 & $0.94-2.12$ & $0.092^{2}$ & 1.43 & $0.92-2.23$ & 0.115 \\
\hline Total & 604 & & & & & & & \\
\hline
\end{tabular}

Lingering grief outcomes by study group, stratified by sex Do you still feel very sad when you think about your loss?

\begin{tabular}{|c|c|c|c|c|c|c|c|c|}
\hline \multicolumn{3}{|l|}{ Females } & \multicolumn{3}{|c|}{ Unadjusted } & \multicolumn{3}{|c|}{ Full model } \\
\hline Exposure & $\mathbf{n}$ & $\%$ yes & OR & $95 \% \mathrm{Cl}$ & p-value & OR & $95 \% \mathrm{Cl}$ & p-value \\
\hline Comparison & 188 & 83.0 & 1.00 & & & 1.00 & & \\
\hline Camp only & 93 & 84.9 & 1.16 & $0.58-2.29$ & 0.675 & 1.09 & $0.48-2.48$ & 0.845 \\
\hline Leader & 34 & 91.2 & 2.12 & $0.61-7.40$ & 0.237 & 2.03 & $0.49-8.38$ & 0.326 \\
\hline Community PSS & 115 & 87.8 & 1.48 & $0.75-2.91$ & 0.256 & 1.22 & $0.58-2.56$ & 0.594 \\
\hline Total & 430 & & & & & & & \\
\hline \multicolumn{9}{|l|}{ Males } \\
\hline \multicolumn{9}{|l|}{ Exposure } \\
\hline Comparison & 155 & 79.4 & 1.00 & & & 1.00 & & \\
\hline Camp only & 97 & 81.4 & 1.14 & $0.60-2.17$ & 0.686 & 0.73 & $0.34-1.60$ & 0.436 \\
\hline Leader & 32 & 78.1 & 0.93 & $0.37-2.34$ & 0.876 & 0.57 & $0.18-1.78$ & 0.332 \\
\hline Community PSS & 78 & 82.1 & 1.19 & $0.59-2.39$ & 0.626 & 1.06 & $0.67-1.69$ & 0.809 \\
\hline Total & 362 & & & & & & & \\
\hline
\end{tabular}




\section{Hgrizons}

Lingering grief outcomes by study group, stratified by sex (continued) Do you still feel very angry when you think about your loss?

Females

Exposure

Comparison

Camp only

Leader

Community PSS

Total

Males

Exposure

Comparison

Camp only

Leader

Community PSS

Total
Unadjusted

95\% Cl p-value

1.00

0.81-2.23 0.261

0.55-2.42 0.705

$0.87-2.26 \quad 0.163$

63.5

1.40

430

(n)

\begin{tabular}{|c|c|c|c|c|c|c|c|c|}
\hline \multicolumn{3}{|l|}{ Females } & \multicolumn{3}{|c|}{ Unadjusted } & \multicolumn{3}{|c|}{ Full model } \\
\hline Exposure & $\mathrm{n}$ & $\%$ yes & OR & $95 \% \mathrm{Cl}$ & p-value & OR & $95 \% \mathrm{Cl}$ & $p$-value \\
\hline Comparison & 188 & 41.0 & 1.00 & & & 1.00 & & \\
\hline Camp only & 93 & 33.3 & 0.72 & $0.43-1.21$ & 0.217 & 0.59 & $0.32-1.11$ & 0.101 \\
\hline Leader & 34 & 26.5 & 0.52 & $0.23-1.17$ & 0.115 & 0.43 & $0.17-1.13$ & $0.088^{2}$ \\
\hline Community PSS & 115 & 59.1 & 2.09 & $1.30-3.34$ & $0.002^{1}$ & 1.71 & $1.01-2.90$ & $0.046^{1}$ \\
\hline Total & 430 & & & & & & & \\
\hline \multicolumn{9}{|l|}{ Males } \\
\hline \multicolumn{9}{|l|}{ Exposure } \\
\hline Comparison & 155 & 38.1 & 1.00 & & & 1.00 & & \\
\hline Camp only & 97 & 37.1 & 0.96 & $0.57-1.62$ & 0.880 & 0.76 & $0.40-1.44$ & 0.397 \\
\hline Leader & 32 & 31.3 & 0.74 & $0.33-1.67$ & 0.468 & 0.59 & $0.22-1.59$ & 0.297 \\
\hline Community PSS & 78 & 41.0 & 1.13 & $0.65-1.97$ & 0.662 & 0.98 & $0.51-1.87$ & 0.951 \\
\hline Total & 362 & & & & & & & \\
\hline
\end{tabular}

${ }_{2}^{1} p<0.05$

${ }^{2} p<0.10$ 


\section{Hgrizons}

Horizons is a global operations research program designed to:

- Identify and test potential strategies to improve HIV/AIDS prevention, care, and support programs and service delivery.

- Disseminate best practices and utilize findings with a view toward scaling up successful interventions.

\section{(2) Population Council}

Horizons is implemented by the Population Council in collaboration with

- International Center for Research on Women (ICRW)

- International HIV/AIDS Alliance

- PATH

- Tulane University

- Family Health International (FHI)

- Johns Hopkins University

For more information, please contact:

Horizons Program, Communications Unit 4301 Connecticut Avenue, NW Suite 280 Washington, DC 20008 USA

Tel: 202-237-9400

Fax: 202-237-8410

Email: horizons@pcdc.org www.popcouncil.org/horizons 